\title{
Improving wood properties for wood utilization through multi-omics integration in lignin biosynthesis
}

\author{
Jack P. Wang1,2, Megan L. Matthews ${ }^{3}$, Cranos M. Williams ${ }^{3}$, Rui Shi' ${ }^{2}$, Chenmin Yang ${ }^{2}$,
} Sermsawat Tunlaya-Anukit², Hsi-Chuan Chen², Quanzi Li ${ }^{4}$, Jie Liu² ${ }^{2}$ Chien-Yuan Lin ${ }^{2,5}$, Punith Naik ${ }^{6}$, Ying-Hsuan Sun ${ }^{7}$, Philip L. Loziuk ${ }^{8}$, Ting-Feng Yeh ${ }^{9}$, Hoon Kim (10 ${ }^{10}$, Erica Gjersing ${ }^{11}$, Todd Shollenberger ${ }^{11}$, Christopher M. Shuford ${ }^{8}$, Jina Song (D) $^{3}$, Zachary Miller ${ }^{12}$, Yung-Yun Huang ${ }^{13}$, Charles W. Edmunds ${ }^{12}$, Baoguang Liu ${ }^{14}$, Yi Sun ${ }^{1}$, Ying-Chung Jimmy Lin'1,2,15, Wei Li,2, Hao Chen², Ilona Peszlen ${ }^{12}$, Joel J. Ducoste ${ }^{6}$, John Ralph (10) ${ }^{10}$, Hou-Min Chang ${ }^{12}$, David C. Muddiman ${ }^{8}$, Mark F. Davis (1) ${ }^{5}$, Chris Smith ${ }^{16}$, Fikret Isik ${ }^{17}$, Ronald Sederoff ${ }^{2} \&$ Vincent L. Chiang ${ }^{1,2,12}$

A multi-omics quantitative integrative analysis of lignin biosynthesis can advance the strategic engineering of wood for timber, pulp, and biofuels. Lignin is polymerized from three monomers (monolignols) produced by a grid-like pathway. The pathway in wood formation of Populus trichocarpa has at least 21 genes, encoding enzymes that mediate 37 reactions on 24 metabolites, leading to lignin and affecting wood properties. We perturb these 21 pathway genes and integrate transcriptomic, proteomic, fluxomic and phenomic data from 221 lines selected from 2000 transgenics (6-month-old). The integrative analysis estimates how changing expression of pathway gene or gene combination affects protein abundance, metabolic-flux, metabolite concentrations, and 25 wood traits, including lignin, tree-growth, density, strength, and saccharification. The analysis then predicts improvements in any of these 25 traits individually or in combinations, through engineering expression of specific monolignol genes. The analysis may lead to greater understanding of other pathways for improved growth and adaptation.

\footnotetext{
${ }^{1}$ State Key Laboratory of Tree Genetics and Breeding, Northeast Forestry University, Harbin 150040, China. ${ }^{2}$ Forest Biotechnology Group, Department of Forestry and Environmental Resources, North Carolina State University, Raleigh, NC 27695, USA. ${ }^{3}$ Electrical and Computer Engineering, North Carolina State University, Raleigh, NC 27695, USA. ${ }^{4}$ State Key Laboratory of Tree Genetics and Breeding, Chinese Academy of Forestry, Beijing 100091 , China. ${ }^{5}$ Joint BioEnergy Institute, Lawrence Berkeley National Laboratory, Emeryville, CA 94608, USA. ${ }^{6}$ Civil, Construction and Environmental Engineering, North Carolina State University, Raleigh, NC 27695, USA. ${ }^{7}$ Department of Forestry, National Chung-Hsing University, Taichung 40227, Taiwan. ${ }^{8}$ Department of Chemistry, W.M. Keck Fourier Transform Mass Spectrometry Laboratory, North Carolina State University, Raleigh, NC 27695, USA. ${ }^{9}$ School of Forestry and Resource Conservation, National Taiwan University, Taipei 10617, Taiwan. ${ }^{10}$ Department of Biochemistry, and the DOE Great Lakes Bioenergy Research Center, Wisconsin Energy Institute, University of Wisconsin, Madison, WI 53726, USA. ${ }^{11}$ National Bioenergy Center, National Renewable Energy Laboratory, Golden, CO 80401, USA. ${ }^{12}$ Department of Forest Biomaterials, North Carolina State University, Raleigh, NC 27695, USA. ${ }^{13}$ Department of Operations Research, North Carolina State University, Raleigh, NC 27695, USA. ${ }^{14}$ Department of Forestry, Beihua University, Jilin 132013, China. ${ }^{15}$ Department of Life Sciences, College of Life Science, National Taiwan University, Taipei 10617, Taiwan. ${ }^{16}$ Bioinformatics Research Center, North Carolina State University, Raleigh, NC 27695, USA. ${ }^{17}$ Department of Forestry and Environmental Resources, North Carolina State University, Raleigh, NC 27695, USA. Correspondence and requests for materials should be addressed to V.L.C. (email: vchiang@ncsu.edu)
} 
W ood has long been used as construction material and for pulp and paper production. Such uses are in considerable part determined by the properties of lignin in wood. Removal of lignin is the key step in pulp and paper production and the conversion of biomass to liquid biofuels. Lignin was discovered in wood by Anselme Payen in 1839 as the incrusting material that must be removed to isolate wood's useful fiber, cellulose ${ }^{1}$. Lignin is a major phenolic polymer in plant secondary cell walls and is formed predominately from the oxidative polymerization of three major monolignols, 4-coumaryl alcohol (H-subunit, 20, Fig. 1), coniferyl alcohol (G-subunit, 22), and sinapyl alcohol (S-subunit, 24) ${ }^{2}$ in angiosperms. The monolignols polymerize around a framework of cellulose and hemicelluloses to form secondary cell walls in trees to make wood. Despite more than half a century of investigation, there is not yet a fundamental quantitative basis for understanding the regulation of lignin biosynthesis and its effects on the properties and utilization of wood.

We now present a quantitative multi-omics integration of the regulation of lignin biosynthesis in wood formation in the model woody plant Populus trichocarpa (Torr. \& Gray) that improves wood properties for utilization. The genome sequence of $P$. trichocarpa $a^{3}$ identified the genes for the biosynthesis of lignin from its monolignol precursors ${ }^{4}$. A core set of 21 genes $^{4}$ (known at the onset of this study) encode specific enzymes that mediates a biosynthetic pathway of 37 metabolic-fluxes converting phenylalanine (1, Fig. 1) through 24 intermediate metabolites into the three monolignols $(20,22 \text {, and } 24)^{5}$ for lignin formation that determines wood properties. Using transgenic P. trichocarpa, we systematically integrated five levels of regulation (genomic, transcriptomic, proteomic, fluxomic, and phenomic, Fig. 1, Fig. 2a, b) to quantify the effects of the expression of monolignol biosynthetic pathway genes on wood properties and wood utilization, such as saccharification (extraction of sugars) (Fig. 2c).

\section{Results}

Overall approach. The five level multi-omics integrative analysis involves three major steps (1-3, Fig. 2b). (1) Quantifying the relationships between the abundances of gene-specific transcripts and proteins. (2) Establishing the effects of gene-specific pathway protein abundances on metabolic-flux and metabolite concentrations. (3) Quantifying the effects of predicted metabolicflux and metabolite concentrations on specific end products (lignin and other wood properties, Fig. 2c). We defined these relationships through xylem-specific transgenic perturbation of the 21 monolignol pathway genes (Fig. 2a, Supplementary Table 1; Supplementary Data 1) to provide a broad range of variation in the levels of gene expression and the downstream responses. Many of these genes are phylogenetically paired members within different gene families ${ }^{4}$. We therefore used RNA interference constructs (types I-III, Supplementary Fig. 1a) to downregulate individual genes, gene-pairs or gene families. Artificial microRNA constructs (type IV, Supplementary Fig. 1a) were also designed to target the specific downregulation of single genes within gene families. We generated $\sim 2000$ transgenic $P$. trichocarpa trees and selected 221 independent lines showing varying degrees of gene downregulation (Supplementary Table 1, Supplementary Data 1, Supplementary Note).

Monolignol biosynthetic genes are abundantly expressed in both fiber and vessel cells (the key wood-forming cells; Supplementary Fig. 1b) in stem-differentiating xylem. We isolated the stem-differentiating xylem from the transgenics and wildtype and analyzed 239 full transcriptomes and 239 proteomes to regress the abundances of transcripts and proteins (Fig. 2a, b). Using recombinant proteins from the 21 monolignol pathway genes used in this study, we determined 207 reaction and inhibition enzyme kinetic parameters to predict the effects of protein abundances on pathway metabolic-fluxes and metabolite concentrations (Fig. 2a, b, Supplementary Data 9) ${ }^{5-8}$. To determine the effects of metabolic-fluxes and metabolite concentrations on lignin and wood properties, we quantified the chemical composition of 220 wood samples, and 76 lignin samples using 2D HSQC NMR for lignin composition and structures (Fig. 2a, b, Supplementary Note describes the number of samples used for each quantification). We measured the growth of 221 lines, the modulus of elasticity (MOE) of 416 wood samples, the density of 213 wood samples, and tested 236 wood samples for saccharification efficiency (Fig. 2a, b, Supplementary Note). All these data were then systematically integrated to describe the transduction of biological information from the 21 monolignol genes through transcripts, proteins, metabolic-fluxes, and metabolite concentrations, leading to specific lignin and wood properties (Fig. 2a-c).

Monolignol gene transcript and protein relationships. We analyzed the whole transcriptomes of the stem-differentiating xylem of 221 transgenics and 18 wildtypes by RNA-seq (GEO accession number: GSE78953) (Supplementary Fig. 1c, d). Transgenic suppression provided broad variation in the levels of monolignol gene expression (Supplementary Fig. 2). Variation in gene expression arose from (1) targeted transgene suppression, (2) indirect (non-targeted) effects of other monolignol transgenes, and (3) seasonal and environmental effects (Supplementary Note, Supplementary Fig. 3). Targeted transgenesis always resulted in a wide range of target gene suppression (Fig. 3a, Supplementary Data 1). For example, the targeted transgenic suppression of PtrPAL1 expression ranged from 18.4 (i8-8-2, Fig. 3a) to $92.5 \%$ (i6-9-3) of the parental wildtype level. PtrPAL1 was also suppressed by homologous transgenes of its family members, such as PtrPAL3 (Fig. 3b), and to a lesser extent, by transgenes of members from other families, such as PtrAldOMT2 (Fig. 3b), through gene interactions. The indirect effect of genetic interactions was observed for many monolignol genes, suggesting unknown higher-order regulation (Fig. 3b). Seasonal and environmental effects in the greenhouse (Supplementary Note) also contributed to variations in transcript abundance, as seen for PtrPAL1 expression in the wildtypes (white bars, Fig. 3a). These variations provided a broad range of gene expression to correlate transcript abundance and protein abundance, and increased the power of the integrative analysis.

We determined the absolute quantities of the monolignol biosynthetic proteins in the stem-differentiating xylem of the 239 transgenics and wildtype using protein cleavage-isotope dilution mass spectrometry (PC-IDMS) and protein-specific stableisotope-labeled internal standards, following Shuford et al. $(2012)^{9}$. Specific reduction of protein abundance was observed in the downregulated transgenics for all monolignol pathway enzymes (Fig. 3b). Protein and transcript abundance for specific monolignol genes showed significant positive linear relationships (Fig. 4, Supplementary Fig. 2, Supplementary Table 2). We assembled a simple linear regression equation for each monolignol gene to describe the efficiency of translation of the transcript to the protein (equations 1-20, Supplementary Data 2). On average, each transcript molecule (inferred from RNA-seq, Supplementary Note) produces a steady-state level of $\sim 1.5 \times 10^{4}$ molecules of the protein ( $\beta_{\mathrm{i}}$ values, Supplementary Table 2$)$. Translation efficiencies are typically $\sim 10^{4}$ in eukaryotes and $\sim 540$ in prokaryotes ${ }^{10,11}$. Different monolignol genes showed dramatically different efficiencies ( $\beta_{\mathrm{i}}$ values, Supplementary Table 2, Supplementary Note). The monolignol gene transcript abundance explained on average $31 \%$ (coefficient of determination, $R^{2}=$ 
$0.31)$ of the variation in protein abundance, which ranged from 53\% (for PtrPAL2) to 3\% (for PtrCCoAOMT3, a poor predictor) (Supplementary Table 2, Supplementary Note). The protein abundances predicted by the linear regressions were used in mass-balance equations to estimate metabolite concentrations and pathway reaction fluxes (Fig. 2b).

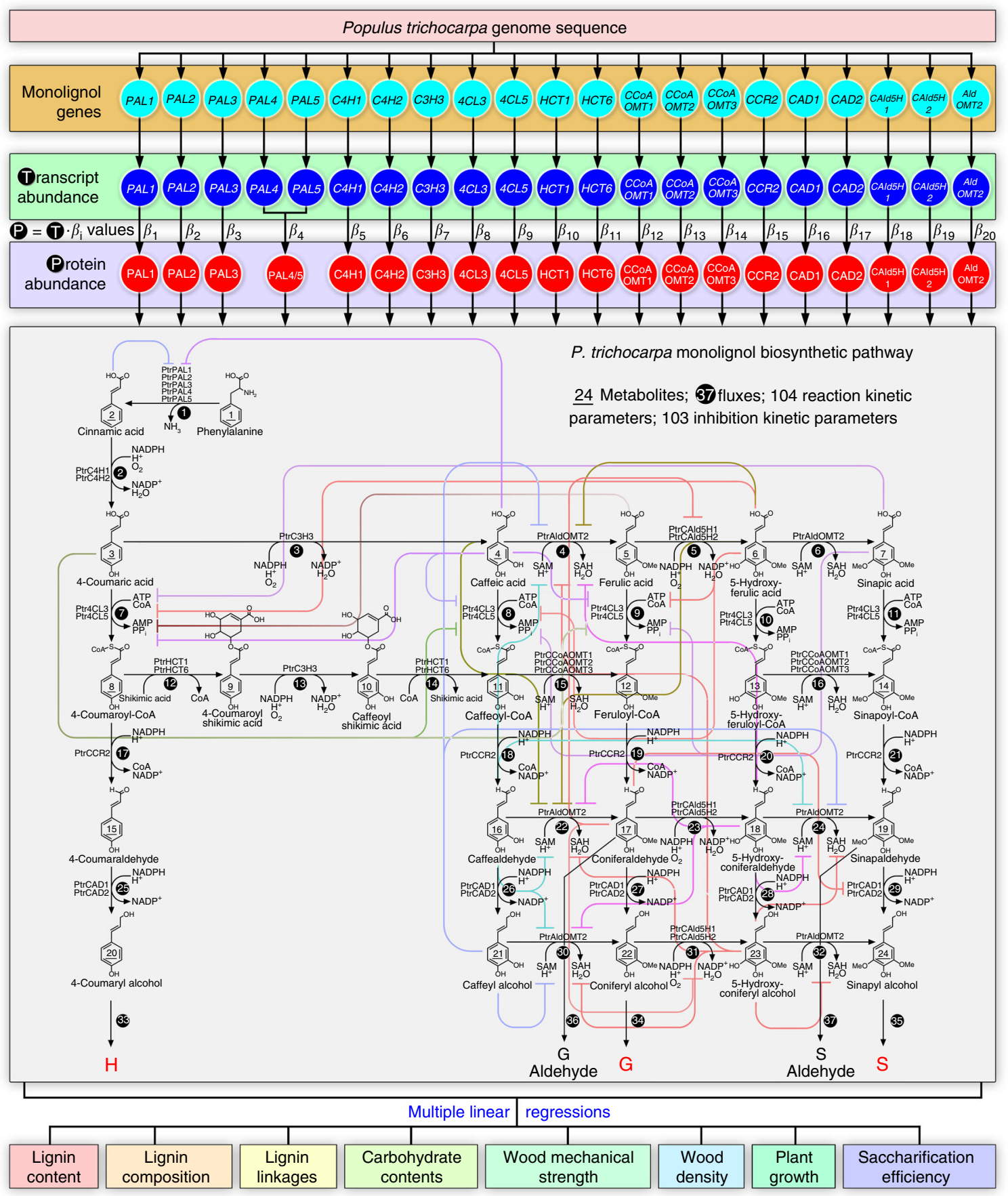

Fig. 1 Our multi-omics integrative analysis of the monolignol biosynthetic pathway in P. trichocarpa predicts wood properties for wood utilization. The pathway is represented by 21 monolignol genes (turquoise circles) identified from the genome sequence, and their corresponding transcripts (blue circles) and proteins (red circles). The gene transcript abundances can be converted to protein abundances by specific $\beta_{\mathrm{i}}$ values from linear regression models (Supplementary Table 2). The monolignol proteins in a metabolic grid convert phenylalanine to monolignols in stem-differentiating xylem. The metabolic grid consists of 24 metabolites (underlined numbers within structures), 37 reaction fluxes (white numbers on black circles), 104 reaction kinetic parameters and 103 inhibition kinetic parameters. Other enzymes, regulators, and components are involved but genes encoding these factors are unknown or not yet sufficiently characterized to be included in this study. Fluxes 16, 20, 21, and 26 were set to zero because kinetic parameters for these reactions are not currently available. Colored lines represent pathway enzyme inhibitions. Multiple linear regressions predict the lignin and wood properties. Abbreviations: Reduced nicotinamide adenine dinucleotide phosphate (NADPH), oxidized nicotinamide adenine dinucleotide phosphate (NADP+), coenzyme A (CoA), adenosine triphosphate (ATP), adenosine monophosphate (AMP), pyrophosphate (PPi), S-adenosylmethionine (SAM), Sadenosylhomocysteine (SAH) 


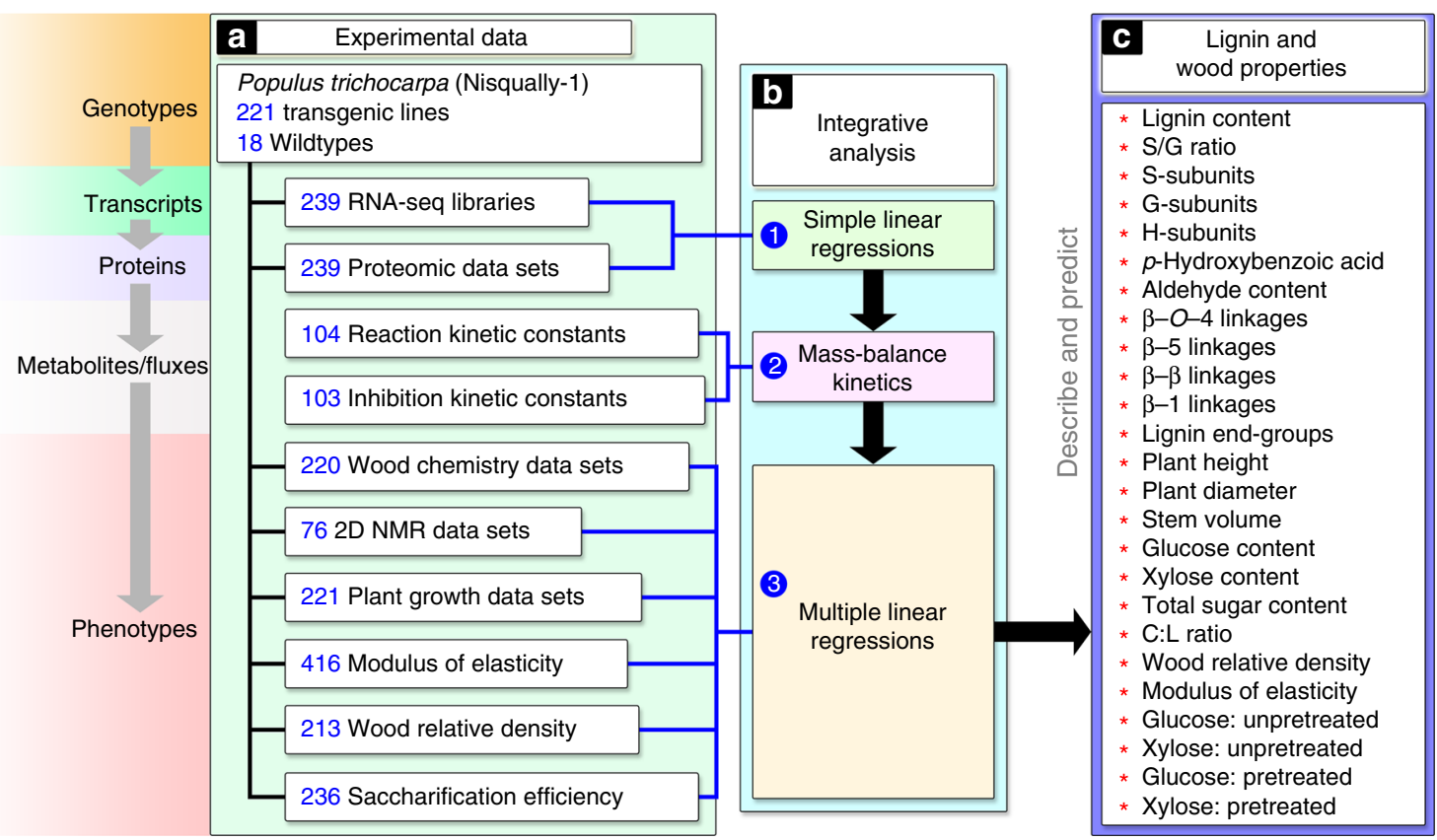

Fig. 2 Systematic integrative analysis of lignin biosynthesis in P. trichocarpa. a The experimental data measured for the transgenics and wildtype. b(1-3) The quantitative relationships between transcript and protein abundances are represented by simple linear regressions. Monolignol pathway metabolites and fluxes are estimated using mass-balance kinetics. Multiple linear regressions associate the metabolite concentrations and fluxes with lignin and wood properties. c The 25 key lignin and wood properties

Metabolic fluxes for monolignol biosynthesis. We previously measured 104 Michaelis-Menten kinetic parameters and 103 inhibition kinetic parameters for purified functional recombinant proteins of 21 monolignol genes ${ }^{5,7}$. These 207 kinetic parameters provide an approximation of the kinetic behaviors of the monolignol enzymes in vivo. We integrated these 207 kinetic parameters and the absolute quantity of monolignol proteins in stem-differentiating xylem into 39 mass-balance equations to predict the effects of changing the abundance of each pathway protein on metabolic-flux, total lignin content, and lignin S/G ratio 5,7 . We now extend these mass-balance equations (equations 21-59, Supplementary Data 2) to include the additional regulation that comes from some specific protein-protein interactions ${ }^{12}$ (Methods) and the transcript/protein regression equations (equations 1-20) to illustrate quantitatively how changes in the pathway gene transcript levels affect protein abundance, metabolite concentrations, metabolic-flux, and additional major lignin properties. These new properties include the abundances of the major lignin subunits (H, G and S) and their structural linkages, as well as two non-monolignol phenolics in lignin, based on semiquantitative two-dimensional nuclear magnetic resonance (2D NMR) of lignin from transgenic trees. The non-monolignol phenolics are p-hydroxybenzoic acid and hydroxycinnamaldehydes (Fig. 2c, Fig. 1), which are usually present in minor quantities in lignin of wildtype $P$. trichocarpa. In transgenics modified in the expression of specific monolignol genes, the abundance of some of these non-monolignol phenolics incorporated into lignin is elevated (Supplementary Note) and affects wood properties (Supplementary Data 3-8).

Our current 59 equations (equations 1-59, Supplementary Data 2) describe how changes in monolignol transcript abundance affect protein abundances and the 37 metabolicfluxes and 24 metabolite concentrations in the pathway (Fig. 1). We next characterized 25 major lignin and wood chemical and physical properties (Fig. 2c; and described in sections below) in the transgenics and wildtype (Supplementary Table 1) for multiple linear regressions (equations 60-84, Supplementary
Data 2, Methods) to predict the effects of pathway flux and metabolite concentrations on these wood properties. The final 84 equations (equations 1-84, Supplementary Data 2) represent the quantitative relationships linking monolignol genes to transcripts, proteins, predicted metabolic-flux, predicted metabolite concentrations, and 25 lignin and wood chemical and physical properties (Fig. 2a-c).

Wood chemical properties. To quantify the effects of monolignol gene perturbations on wood chemical properties, we analyzed stem wood from 203 transgenics and 17 wildtypes. Wildtype wood contains on average $21.7 \%$ lignin, $49.8 \%$ glucose, $16.2 \%$ xylose, $2.8 \%$ mannose, and $1.0 \%$ galactose $(\%=\mathrm{g} / 100 \mathrm{~g}$ dry wood), with a total carbohydrate to lignin ratio (C:L) of 3.2 (Supplementary Data 3). The C:L ratio is a direct indicator of the potential maximum cellulosic yield for wood pulp and maximum sugar yield for biofuels and other bioproducts. The levels of monolignol gene expression significantly altered the wood composition; we observed broad variation of lignin content that ranged from 9.4 to $25.0 \%$ (Fig. 5a). Glucose varied from 37.0 to $66.9 \%$ (Fig. 5b), xylose from 12.4 to $23.9 \%$ (Fig. 5 c), mannose from 0.0 to $4.9 \%$ (Fig. $5 \mathrm{~d}$ ), and galactose from 0.1 to $2.9 \%$ (Fig. 5e, Supplementary Data 3, Supplementary Fig. 4). The C:L ratios of the transgenics ranged from 2.5 to 8.8 (Fig. 5f), demonstrating the feasibility of generating raw materials suitable for a wide range of end uses. Consistent with our previous finding ${ }^{13}$, lignin content showed a significant negative linear relationship with total carbohydrate (Fig. 5g), having a slope of -0.8 and a correlation coefficient of -0.4 . Therefore, for every $1 \%$ reduction in lignin, the total carbohydrate increases on average by $\sim 0.8 \%$ ( $\%=\mathrm{g} / 100 \mathrm{~g}$ dry wood).

Lignin from 68 transgenics and 8 wildtypes was analyzed by $2 \mathrm{D}$ NMR to quantify lignin subunit composition and major interunit linkages (Fig. 6, Supplementary Data 4, Supplementary Fig. 5, Supplementary Fig. 6, Supplementary Note). Changing monolignol gene expression significantly altered lignin composition. In 

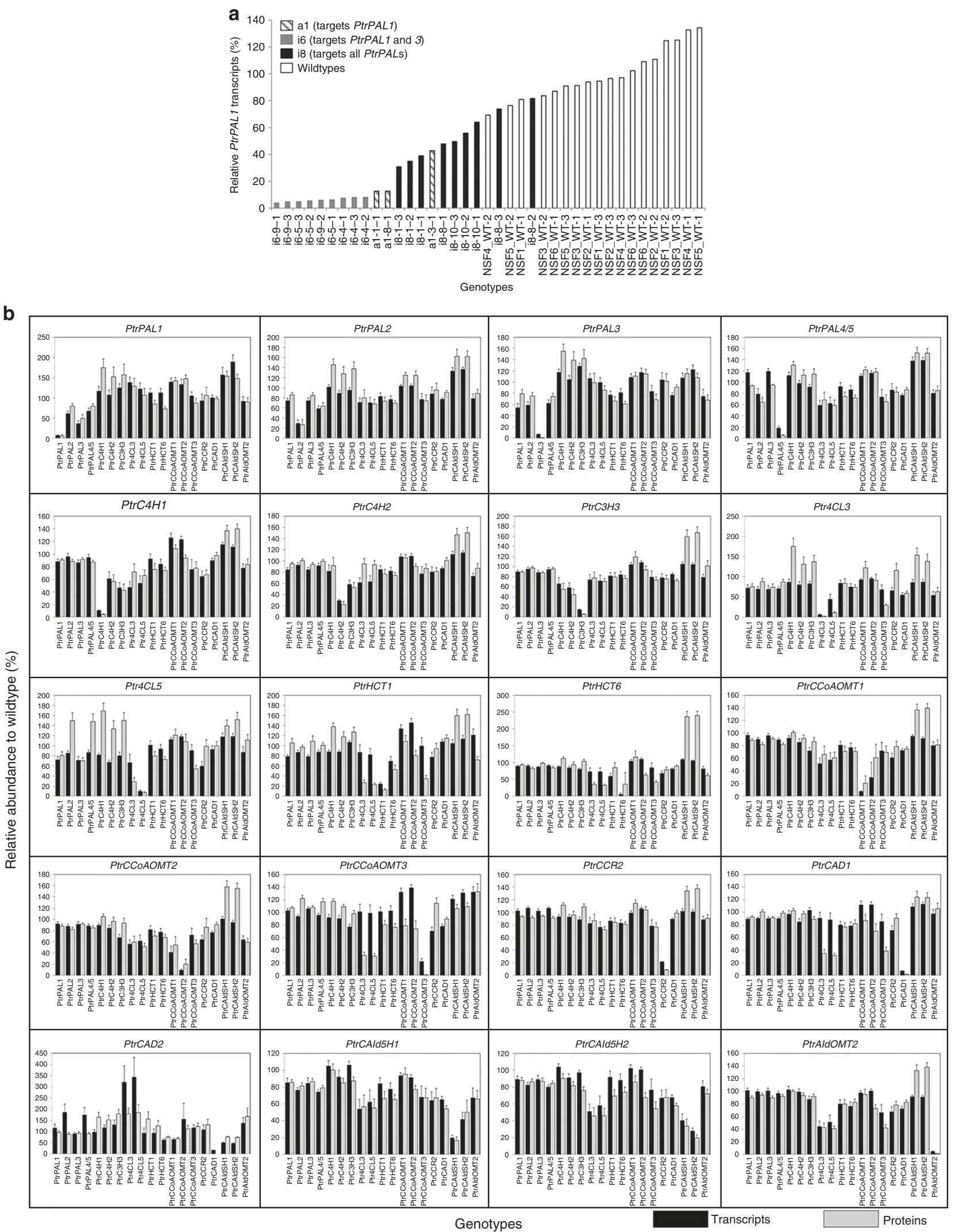

Fig. 3 The abundance of the monolignol biosynthetic gene transcripts and proteins in transgenic $P$. trichocarpa lines. a Relative abundance of PtrPAL1 transcripts in the transgenic and wildtype $P$. trichocarpa. White bars represent PtrPAL1 transcript abundance in wildtype trees. Striped bars represent PtrPAL1 transcript abundance in transgenic lines targeting the downregulation of PtrPAL1. Gray bars represent PtrPAL1 transcript abundance in transgenic lines targeting the downregulation of both PtrPAL1 and PtrPAL3. Black bars represent PtrPAL1 transcript abundance in transgenic lines targeting the downregulation of all five PtrPALs. $\mathbf{b}$ The $x$-axis represents the genotypes of the transgenic lines, and the $y$-axis represents relative transcript (black bars) and protein (gray bars) abundance to the average wildtype level. Error bars represent the standard error of at least three biological replicates 


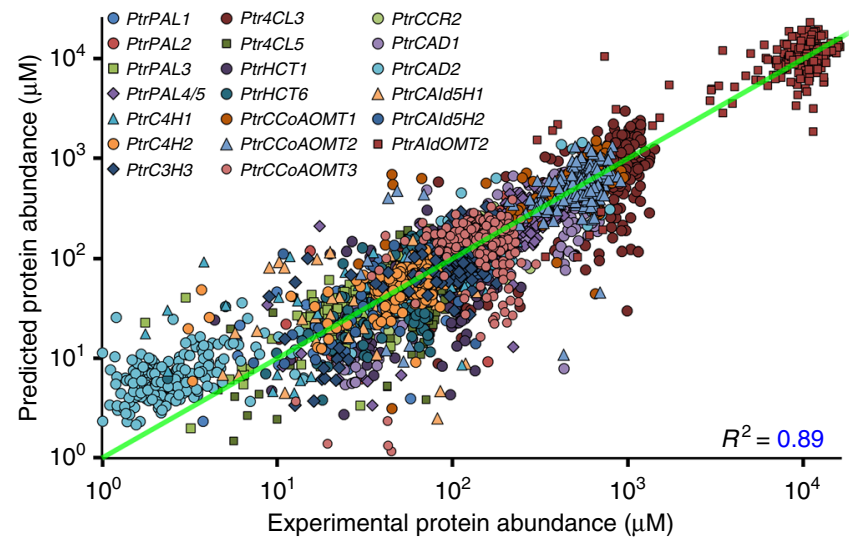

Fig. 4 Regression of monolignol transcript/protein relationships. Transcript abundance is used in the linear regression equations (Supplementary Table 2) to obtain the predicted protein abundance ( $y$-axis), which is regressed on the experimental protein abundance ( $x$-axis). The equations can explain $~ 89 \%$ of the variation in abundance of the monolignol biosynthetic proteins in the 239 transgenic and wildtype samples, based on the abundance of the gene transcripts. Each data point represents the abundance of a monolignol gene for a replicate pool of one wildtype or transgenic line containing 3-5 clonally propagated plants

the lignin of transgenics, S-subunits ranged from 11.0 to $74.8 \%$, G-subunits from 5.1 to $88.8 \%, \mathrm{H}$-subunits from 0.2 to $44.8 \%$, $p$ hydroxybenzoic acid from 1.4 to $14.8 \%$, and $\mathrm{S} / \mathrm{G}$ ratios from 0.12 to 9.9 (Fig. 6b-o, Supplementary Data 4, Supplementary Note). The wide variation in lignin subunit composition also significantly altered the interunit linkages. $\beta$-Ethers $(\beta-O-4$-ether linkages) ranged from 76.9 to $95.9 \%$, phenylcoumarans $(\beta-5)$ from 1.2 to $16.3 \%$, resinols $(\beta-\beta)$ from 1.4 to $12.7 \%$, spirodienones $(\beta-1)$ from 0.0 to $3.5 \%$, and cinnamyl alcohol end-groups from 4.6 to $14.2 \%$ (Supplementary Data 4, Supplementary Note).

Wood physical properties. The consequences of changing monolignol gene expression on plant growth, wood mechanical strength, and density has not previously been systematically investigated in any tree species within a uniform genetic background. We measured the growth (height, diameter, and stem volume) of 221 transgenic and wildtype lines (6-month-old). Plant growth was significantly affected by the downregulation of specific monolignol genes (Supplementary Data 5). Transgenics downregulated in PtrHCT, Ptr4CL and PtrCCR families showed severe growth reduction (Supplementary Data 5) that does not correlate with lignin content. Some transgenics with low lignin, such as PtrPAL transgenics with $9.4 \%$ lignin $(\%=\mathrm{g} / 100 \mathrm{~g}$ dry wood) (i7-2-2, Supplementary Data 3 ) showed growth similar to wildtype (i7-2-2, Supplementary Data 5). Growth is also not associated with lignin subunit composition or specific linkages (Supplementary Data 5), but the growth reduction has been attributed to collapsed vessel elements ${ }^{14}$, transcriptional reprogramming $^{15}$, and accumulation of chemical inhibitors ${ }^{16}$ in some transgenics.

The MOE is a quantitative index of wood mechanical strength and is widely used to guide solid wood and engineered wood utilization. A higher MOE indicates that the wood is stiffer and less prone to deformation. We measured MOE perpendicular to the longitudinal axis for stem segments of 416 transgenic and wildtype trees (Supplementary Data 6). Transgenic trees showed broad variation of MOE that ranged from 355 to 6058 MegaPascals (MPa), compared to the wildtype $(3763 \pm 121 \mathrm{MPa})$ (Supplementary Data 6). MOE is substantially affected by the subunit content and composition of lignin. All transgenic trees with reduced lignin showed proportional reductions in $\mathrm{MOE}$ (Supplementary Data 3 and 6). Under conditions where total lignin content does not change, an increase in hydroxycinnamaldehyde units from 4.0 to $29.2 \%$ in lignin (i33-05, Supplementary Data 4 ) resulted in a $\sim 61 \%$ reduction in MOE (i33-05, Supplementary Data 6).

Wood density (expressed as specific gravity, Supplementary Data 7) is one of the most important properties of wood because of its strong relationship to the yield and quality of wood products ${ }^{17}$. The density of 213 samples of transgenic and wildtype wood varied from 0.26 to 0.43 (wildtype $=0.36$ ) (average density for each line, Supplementary Data 7). Wood density and mechanical strength (MOE) showed a positive correlation (coefficient $=0.57$ ) (Fig. 7a). Each 0.01 unit increase in wood density corresponds to a $125 \mathrm{MPa}$ increase in MOE (Fig. 7a). MOE per 0.01 unit increase in wood density for $P$. cathayana and $P$. tomentosa are 120 and $125 \mathrm{MPa}$, respectively, and averaged $166 \mathrm{MPa}$ across 16 diverse woody plant species ${ }^{18}$.

Saccharification efficiency and lignin. Wood is an attractive resource for sustainable biofuel and biomaterial production. However, the recalcitrant properties of lignin have impeded enzymatic saccharification for biofuels and bioproducts. Acid pretreatment is typically used to reduce lignin recalcitrance to facilitate enzymatic saccharification, but the process is costly and produces enzyme inhibitors ${ }^{19}$. Lowering lignin content reduces or eliminates the need for chemical pretreatment ${ }^{20}$. The saccharification efficiency of 236 wood samples of the transgenics and wildtype generated here was calculated from the quantities of glucose and xylose (the two main sugars in hardwood species) released from unpretreated or pretreated (Methods) wood samples (Fig. 7b-f). In unpretreated wildtypes, the saccharification efficiency of releasing glucose from glucans (cellulose and glucomannan) is $24.8 \%$, and xylose from xylans is $8.3 \%$ (Fig. $7 \mathrm{c}$, d, Supplementary Data 8). Unpretreated transgenic wood with reduced lignin showed high glucan saccharification efficiencies, up to $87.2 \%$ (Fig. $7 \mathrm{c}$ ) and xylan up to $68.8 \%$ (Fig. $7 \mathrm{~d}$, Supplementary Data 8$)$. Without pretreatment, every $1 \%(\%=\mathrm{g} /$ $100 \mathrm{~g}$ dry wood) lignin content reduction results in a $3.5 \%$ increase in glucose saccharification efficiency (Fig. $7 \mathrm{c}$ ) and a $2.8 \%$ increase in xylose efficiency (Fig. 7d). Pretreatment elevated the saccharification efficiencies of wildtype wood from 24.8 to $47.6 \%$ for glucose (Fig. 7e) and from 8.3 to $52.8 \%$ for xylose (Fig. $7 \mathrm{f}$ ). Pretreatment together with transgenic reduction in lignin content further elevated wood saccharification efficiencies from 47.6 to $91.1 \%$ for glucose (Fig. 7e) and from 52.8 to $99.6 \%$ for xylose (Fig. 7f). The glucan and xylan saccharification efficiencies are strongly and negatively affected by lignin content whether the wood is pretreated or not (Fig. $7 \mathrm{~b}-\mathrm{f}$ ), consistent with previous studies in alfalfa $^{21}$ and Arabidopsis ${ }^{22}$. The efficiency in $P$. trichocarpa is not significantly affected by the lignin subunit composition or its linkage distribution ( $p>0.01$, Fig. 7b). Lignin and sugar composition have been shown to influence saccharification efficiency in Arabidopsis ${ }^{22}$ and unpretreated alfalfa ${ }^{21}$.

Integrative systems analysis. We then systematically combined the individual quantitative effects of changing monolignol gene expression on protein quantity, predicted metabolic-flux, predicted metabolite concentration, lignin (content, subunit composition, and linkages), carbohydrates, growth, MOE, wood density, and saccharification efficiency for an integrative analysis (Fig. 2b). The analysis integrates 84 equations (Supplementary Datas 2 and 9, Methods), describing and predicting the behavior of the lignin biosynthetic pathway and the consequence of 
a

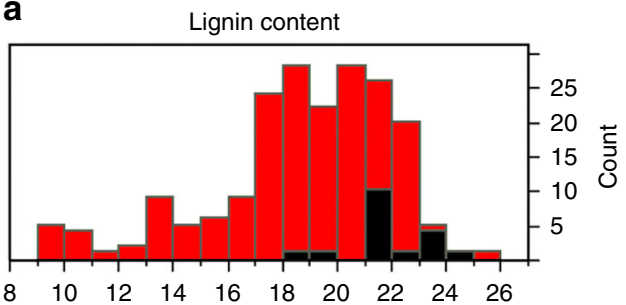

C

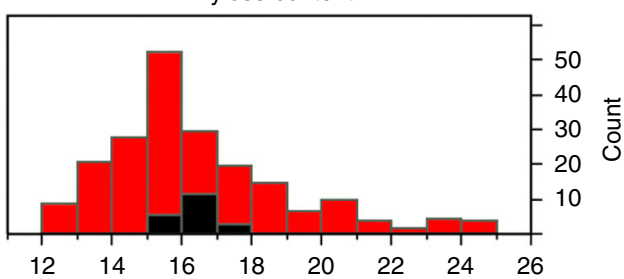

b

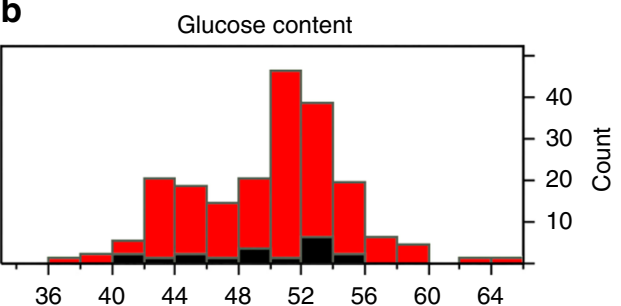

d

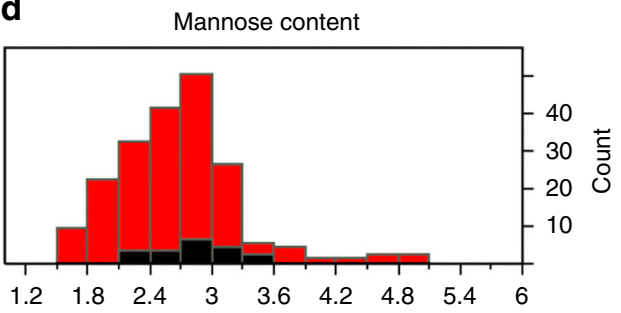

e

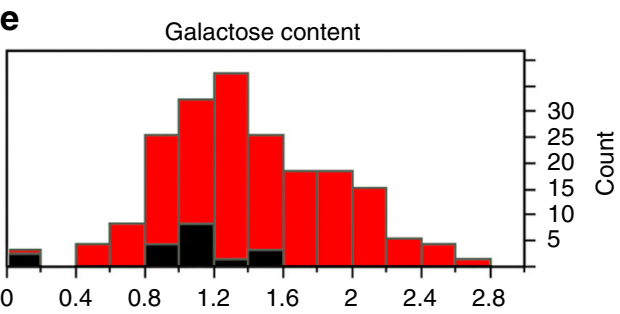

f
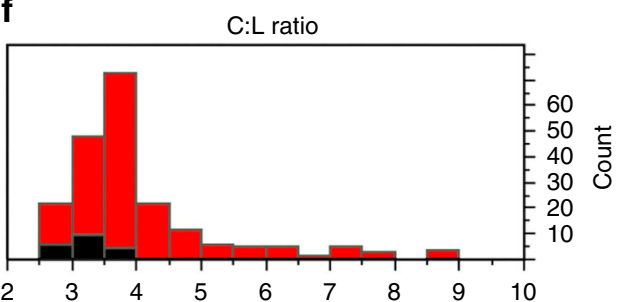

$\%$ Weight of dry wood

203 transgenics

17 wildtypes

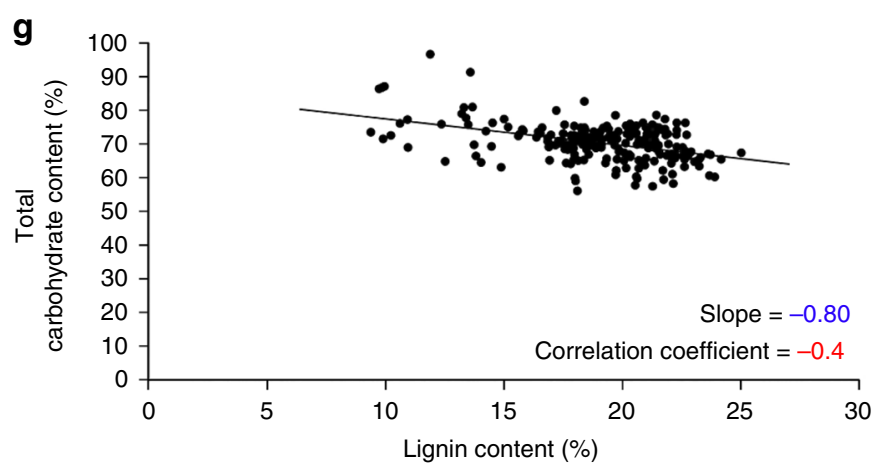

Fig. 5 Variation of lignin and carbohydrate contents in transgenic and wildtype $P$. trichocarpa. a lignin, b glucose, c xylose, $\mathbf{d}$ mannose, and e galactose contents, and the carbohydrate to lignin (C:L) ratio $\mathbf{f}$ in the wood of transgenic (red bars) and wildtype (black bars) P. trichocarpa. See Supplementary Data 3. $\mathbf{g}$ Lignin and total carbohydrate contents are negatively correlated. The negative linear relationship has a slope of -0.8 and a correlation coefficient of -0.4 . Each data point represents a replicate pool of one wildtype or transgenic line containing 3-5 clonally propagated trees

monolignol gene perturbations on the chemical and physical properties of wood. We validated the predictive capabilities of the analysis using five-fold cross-validation. The cross-validation includes re-estimation of all transcript/protein constants $\left(\beta_{\mathrm{i}}\right.$ values) and multiple linear regression parameters in the absence of the validation data sets, thereby providing a validity check of the entire integrative analysis. The training data sets and the validation data sets (Fig. 8a) show similar $R^{2}$ values, indicating that the integrative analysis equations are not overfitting the experimental data. With only transcript abundance as the input, the validated integrative analysis accurately captured the variation in lignin and wood properties of the transgenics and wildtype (Fig. 9). The integrative analysis explained on average $82 \%$ of the variation in lignin properties (Fig. $9 \mathrm{a}-\mathrm{m}$ ) and $72 \%$ for all 25 wood properties (Fig. 9a-y). Lower variations $\left(R^{2}\right.$ of 0.40 and 0.44 , respectively) in glucose and total carbohydrates (Fig. 9q, s) were explained, indicating that these traits are more strongly regulated by mechanisms other than lignin biosynthesis. The analysis predictions and the experimentally measured values showed minimal bias (average bias $=0.96$, Supplementary Table 3 ), suggesting that our integrative analysis has high predictive power. Quantitative estimation of the monolignol pathway fluxes and metabolite concentrations is necessary for the multiple linear regression equations to accurately predict lignin and wood properties (Fig. 8b, Supplementary Note).

Strategic engineering of wood properties. Our integrative analysis (84 integrated equations, Supplementary Data 2) can guide the strategic engineering of wood for better timber and more 

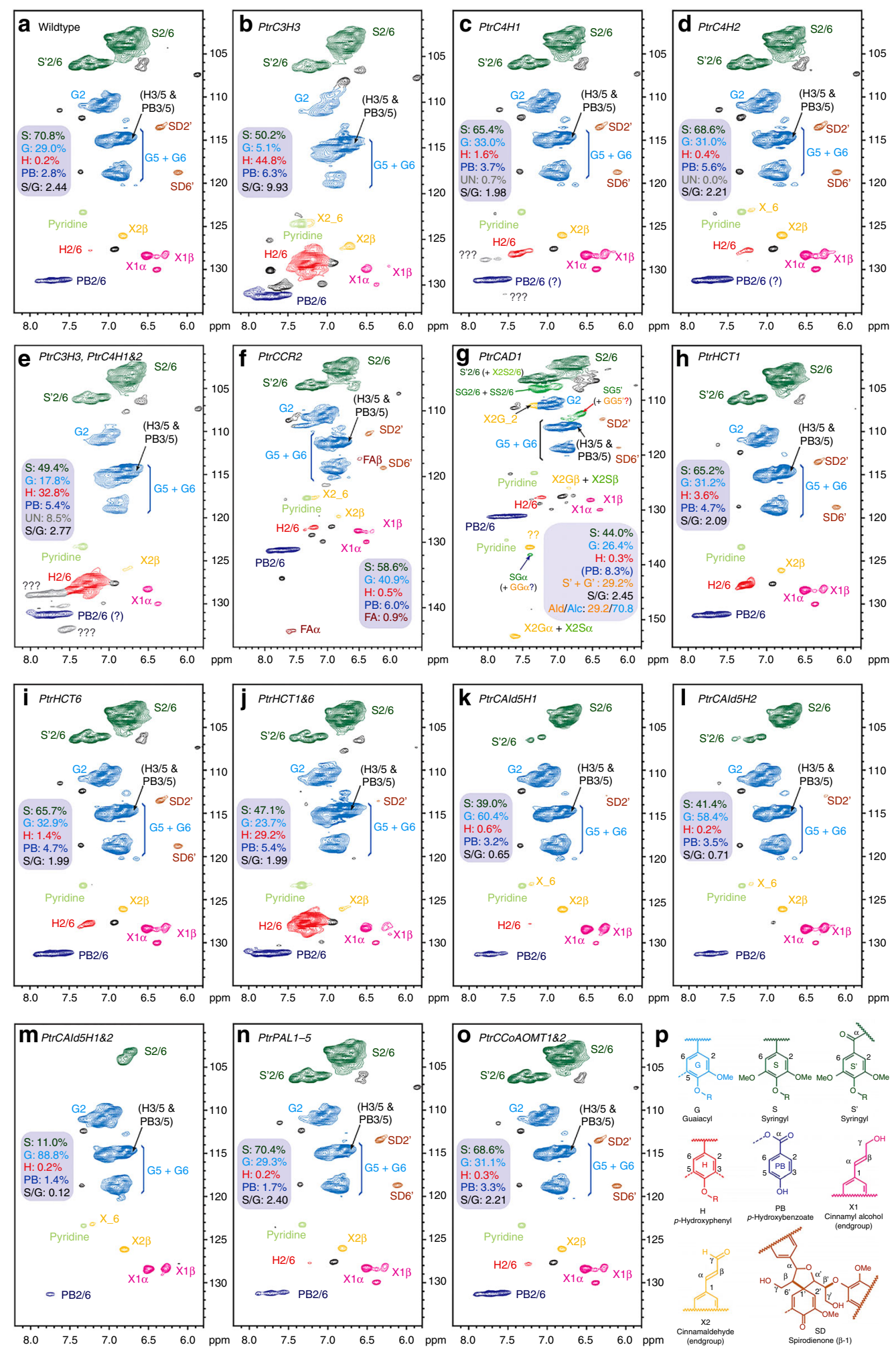

Fig. 6 Composition of lignin quantified by $2 \mathrm{D}$ NMR. Partial (aromatic and double-bond regions) short-range $2 \mathrm{D}{ }^{1} \mathrm{H}-{ }^{13} \mathrm{C}$ correlation $(\mathrm{HSQC}$ ) spectra of enzyme lignin from wildtype (a), and an example of a transgenic $P$. trichocarpa line for each construct downregulated in the gene expression of $\mathbf{b}$ PtrC3H3, c $P \operatorname{trC} 4 H 1$, d $P \operatorname{trC} 4 \mathrm{H} 2$, e PtrC3H3 and PtrC4H1\&2, f PtrCCR2, g PtrCAD1, h PtrHCT1, i PtrHCT6, j PtrHCT1\&6, k PtrCAld5H1, I PtrCAld5H2, m PtrCAld5H1\&2,

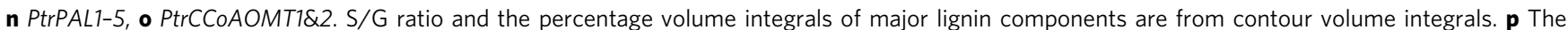
chemical structures of the lignin monomeric subunits, color-coded to match their signal assignments in the spectra. See Supplementary Data 4 for all 2D NMR data 


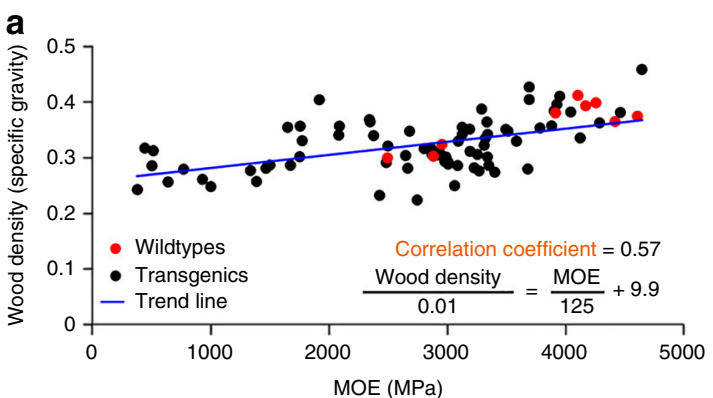

b
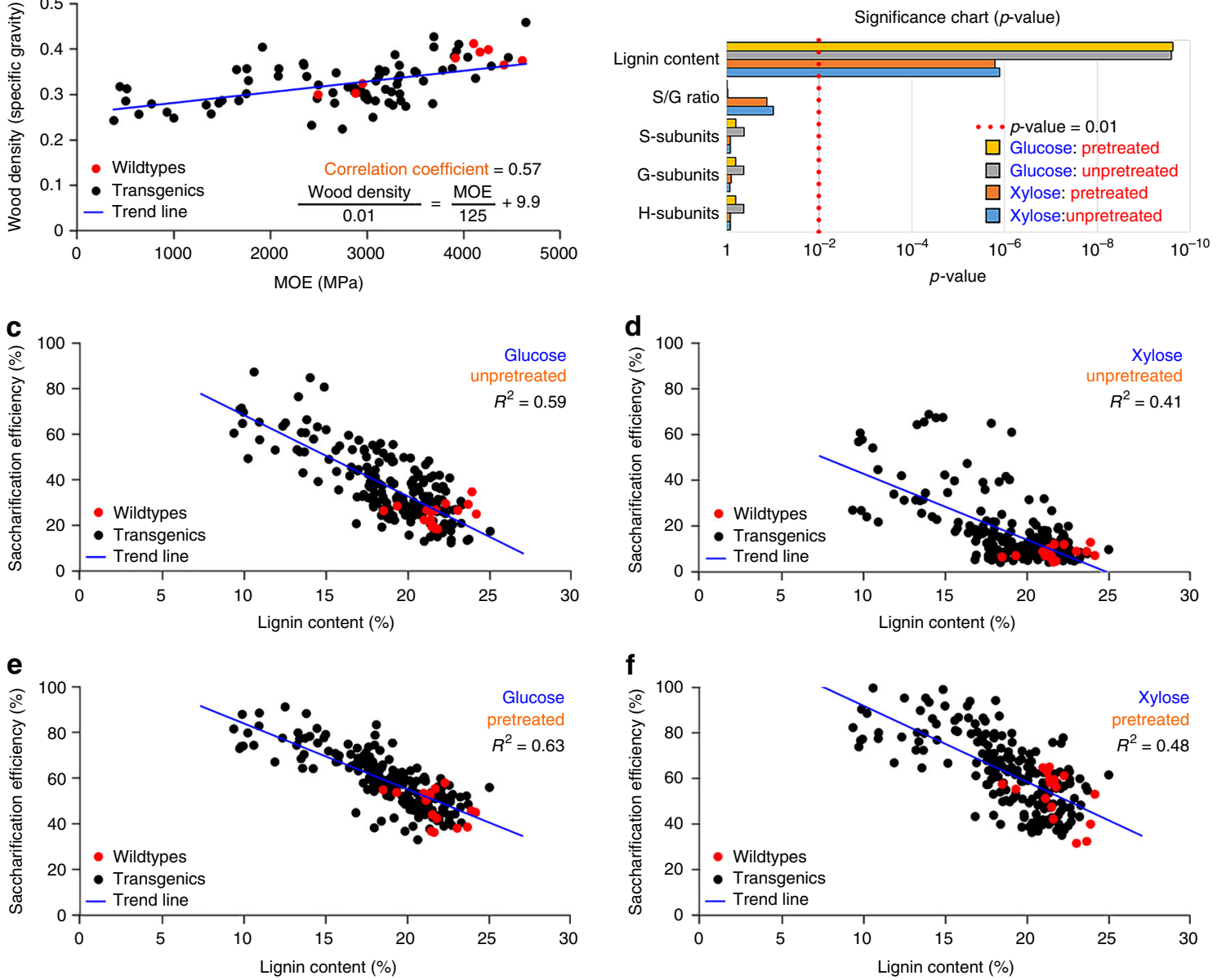

Fig. 7 Quantitative relationships between wood relative density, MOE, lignin content, and saccharification efficiency. a The relationship between experimentally measured wood density and MOE. Each dot represents a replicate pool of one wildtype (red dots) or transgenic (black dots) line containing 3-5 clonally propagated plants. MOE and wood density show a positive linear relationship with a correlation coefficient of 0.57 . Linear regression shows that each 0.01 increase in wood density corresponds to a $125 \mathrm{MPa}$ increase in MOE. b Significance chart ( $p$-value) shows that among the lignin properties, lignin content is the only significant effector $(p<0.01)$ of wood saccharification efficiency. The five highest $p$-values and the corresponding lignin properties are shown. c-f Wood saccharification efficiency is negatively correlated with lignin content. Scatterplots show the percentage release of glucose (c, e) and xylose (d, f) in the transgenic (black dots) and wildtype (red dots) P. trichocarpa. The wood samples were either unpretreated (c, d) or hot-water pretreated $(\mathbf{e}, \mathbf{f})$ (Methods). Each dot represents a replicate pool of one wildtype or transgenic line containing 3-5 clonally propagated plants. $R^{2}$ denotes the coefficient of determination (percent variance explained by the regression)

efficient conversion to pulp and liquid biofuels. High quality timber is dense, strong, and resistant to pests and pathogens, whereas wood pulp and saccharification benefit from reduced wood recalcitrance and increased carbohydrate content. To understand the extent and direction to which specific monolignol genes or combinations of these genes affect lignin and other wood properties, we used the integrative analysis to predict the consequences of varying transcript abundance of a single monolignol gene or a gene family, while all other gene transcripts remained at wildtype levels (Fig. 10, Supplementary Data 10). Different genes and gene families show distinct and specific effects on lignin and wood properties (Fig. 10a, Supplementary Data 10, Supplementary Table 4, Supplementary Note). The effects of changing the expression of an entire gene family are greater than the effects of changing the expression of individual genes within a family (Supplementary Data 10), which indicates that individual family members are functionally redundant and maintain wildtype levels of lignin and wood formation.

Our integrative analysis can be used to identify the best approach for improving single or combinations of multiple lignin and wood properties, while minimizing negative effects on growth. For example, we identified perturbation approaches for a maximal increase in three key properties, i.e., wood density, saccharification efficiency, and the ratio of carbohydrate to lignin (C:L), for more efficient biofuel and pulp/paper production, without significant impact on growth. We analyzed all possible combinations of monolignol gene perturbations, where each gene family was either upregulated, downregulated or remained at wildtype level (Methods). We then ranked these perturbations by the extent of improvement over wildtype for their predicted lignin and wood properties. For a single gene family perturbation, the integrative analysis predicts that the downregulation of $P A L$ delivers the best overall improvement in wood quality. Compared to wildtype, $P A L$ downregulation by $95 \%$ is predicted to exhibit $\sim 70 \%$ increase in glucose release and $\sim 260 \%$ increase in xylose release during saccharification, and $\sim 60 \%$ increase in the C:L ratio (Supplementary Data 10). To achieve maximal improvement in all three key properties simultaneously, perturbation of multiple gene families is needed. Downregulation of both $P A L$ and CCoAOMT genes by $95 \%$ (Fig. 10b) is predicted to increase wood density by $\sim 53 \%$ over wildtype, with $\sim 75$ and $~ 220 \%$ increase in glucose and xylose release, respectively, and $\sim 60 \%$ increase in the 


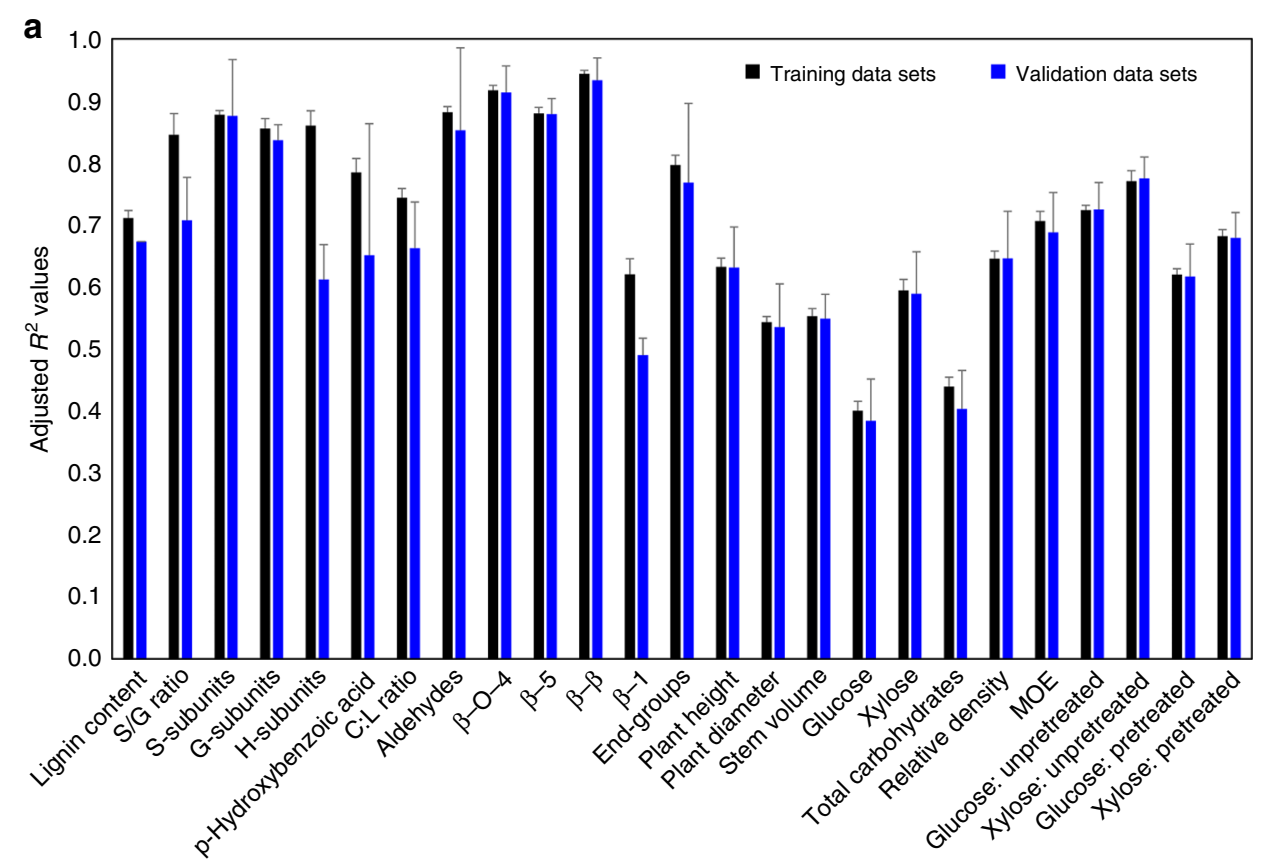

25 key lignin and wood properties

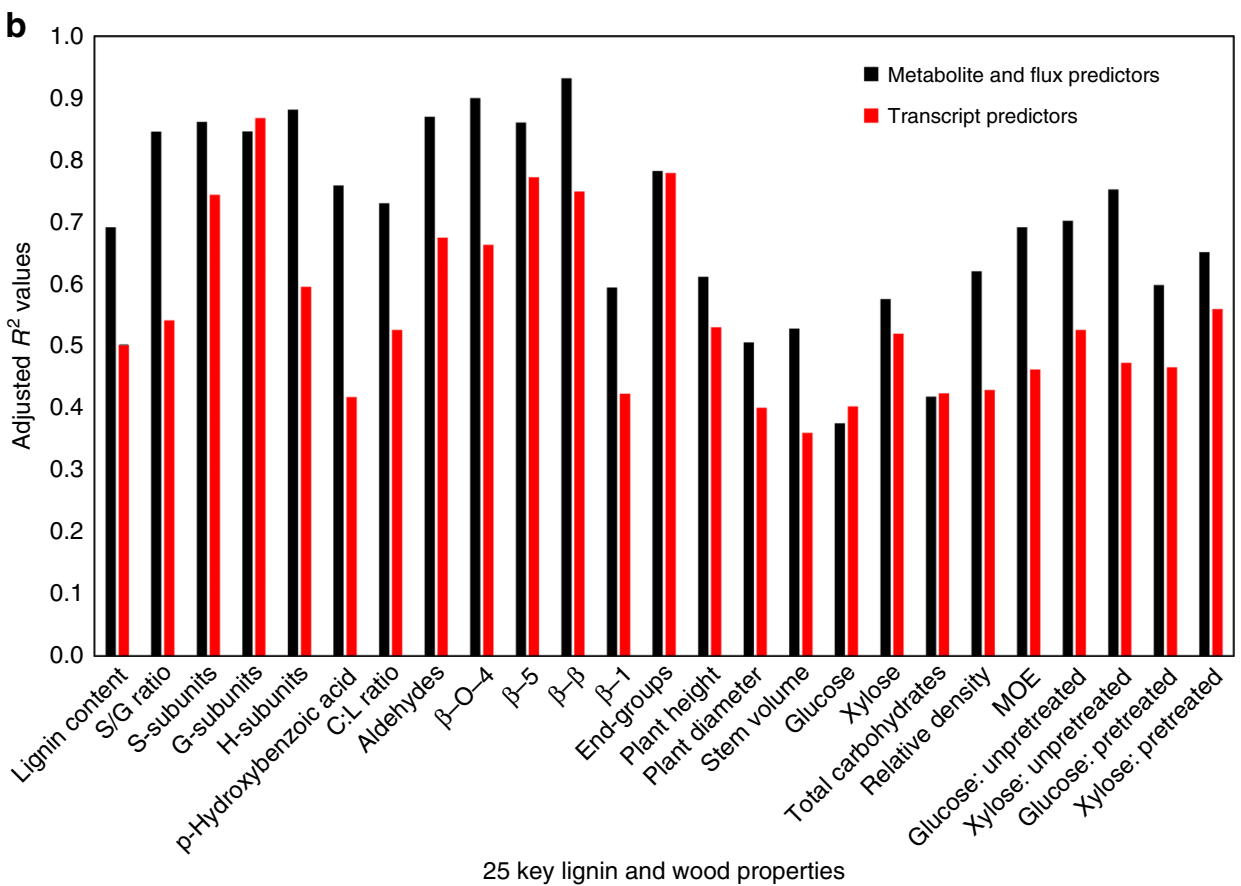

Fig. 8 Integrative analysis validation. a Average adjusted $R^{2}$ values for the training data sets (black bars) and the validation data sets (blue bars) for a fivefold cross-validation. Error bars denote the standard error of five sets of validations. $\mathbf{b}$ Adjusted $R^{2}$ values for the lignin and wood properties predicted using either absolute transcript abundances as predictors (red bars) or metabolic-fluxes and metabolite concentrations as predictors (black bars). For both regression equations, an all-possible-model algorithm was used to determine the specific predictor variables. See Methods for detail. Adjusted $R^{2}$ values represent variation explained by the regressions after adjusting for a different number of predictors

C:L ratio (Fig. 10b). Downregulation of $P A L, C 3 H$ and CCoAOMT gene families is predicted to yield the best overall wood quality (Fig. 10c), with $\sim 41 \%$ increase in wood density, $\sim 153$ and $\sim 456 \%$ increase in glucose and xylose release, respectively, and $\sim 140 \%$ increase in the C:L ratio (Fig. 10c).

Our integrative analysis provides a foundation for future work to incorporate additional regulatory processes that affect lignin and wood properties. For example, epigenetic and transcriptional regulation of monolignol gene expression could be incorporated to understand how developmental (e.g., G-lignin and S-lignin cell-types) and genetic variation affect these properties. Novel pathway components, such as the recently discovered caffeoyl shikimate esterase ${ }^{23-25}$, could also be incorporated and may further increase predictive power. To develop the designed transgenic trees with predicted wood and growth properties for utilization, field trials are essential to validate their viability. Field data on key growth and development regulations linking lignin and wood formation with environmental effects (i.e., biotic and 


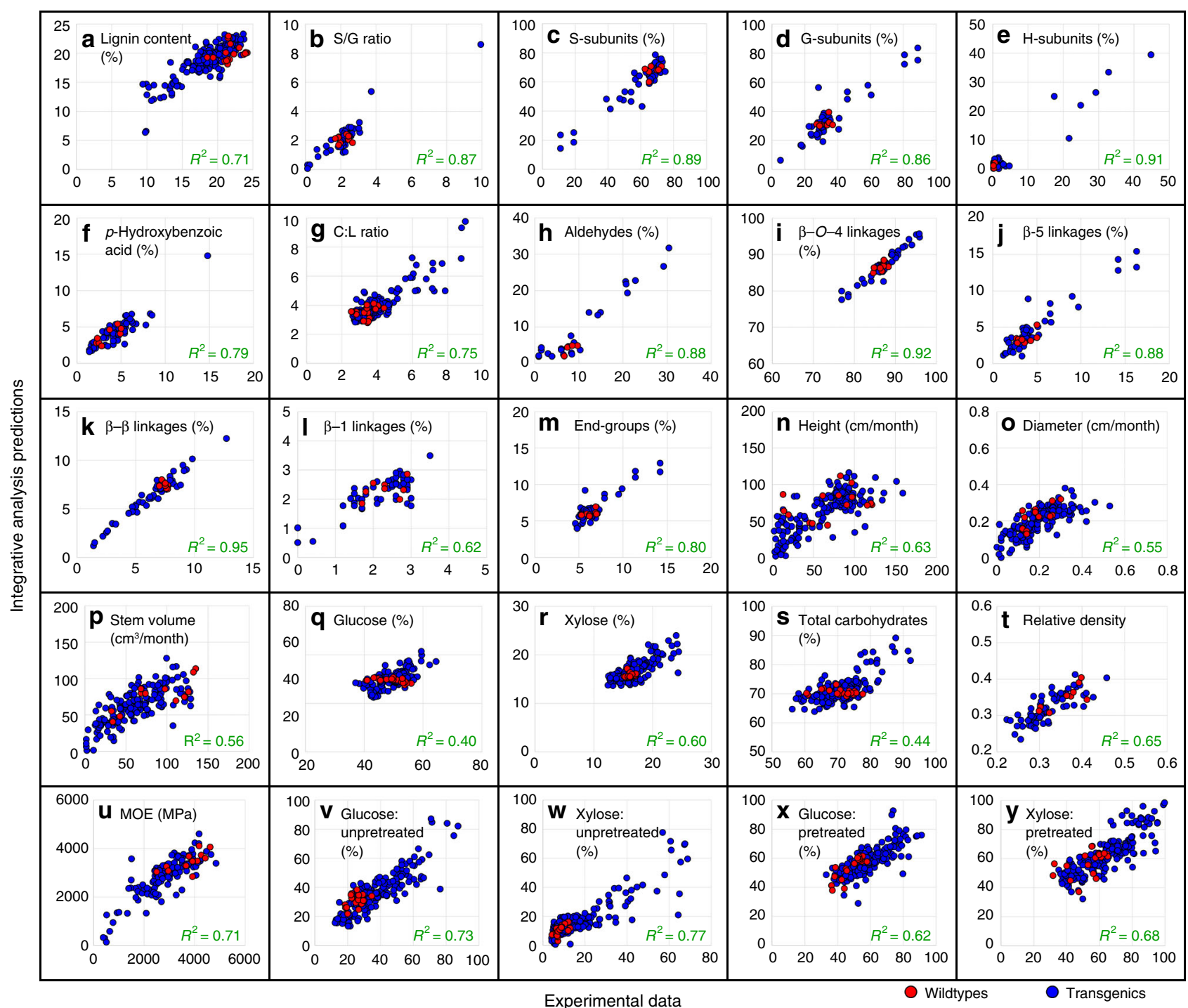

Fig. 9 Scatterplots of the phenotypic variations captured by the lignin-based integrative analysis. The monolignol transcript abundance was used as the only input for the multi-level integrative analysis to predict the corresponding protein abundance, predicted metabolic-flux, predicted metabolite concentrations, and the 25 lignin and wood properties. The figure shows that transcript abundance is used to predict the 25 lignin and wood properties ( $y$-axis, through the multi-level analysis), which are regressed on the experimental data ( $x$-axis, measured in transgenics and wildtype). a Lignin content; b S/G ratio; c S-subunits; d G-subunits; e H-subunits; $\mathbf{f} p$-Hydroxybenzoic acid; $\mathbf{g}$ Carbohydrate to lignin ( $\mathrm{C}: \mathrm{L}$ ) ratio; $\mathbf{h}$ Aldehyde content; i $\beta$-O-4 linkages; j $\beta$-5 linkages; $\mathbf{k} \beta$ - $\beta$ linkages; I $\beta$-1 linkages; $\mathbf{m}$ Cinnamyl alcohol end-groups; $\mathbf{n}$ Height; o Diameter; $\mathbf{p}$ Stem volume; $\mathbf{q}$ Glucose content; $\mathbf{r}$ Xylose content; $\mathbf{s}$ Total carbohydrates; $\mathbf{t}$ Relative density; $\mathbf{u}$ MOE; $\mathbf{v}-\mathbf{y}$ Saccharification efficiency for glucose $(\mathbf{v}, \mathbf{x})$ and xylose (w, $\mathbf{y})$ release from unpretreated ( $\mathbf{v}, \mathbf{w})$ or pretreated $(\mathbf{x}, \mathbf{y})$ wood samples. The $R^{2}$ values are coefficients of determination, showing the percent variation in dependent variables explained by monolignol gene transcript abundance as the predictor. Each data point represents a replicate pool of one wildtype or transgenic line containing 3-5 clonally propagated trees

abiotic stresses) could then be integrated into our analysis for a more comprehensive strategic engineering of wood properties.

\section{Discussion}

Genetic perturbation of monolignol biosynthetic pathway expands the range of variation in monolignol gene transcripts, lignin and wood traits, and saccharification efficiencies, compared to a natural population of $P$. trichocarpa $a^{26,27}$. Sufficient downregulation of any monolignol gene family can modify the properties of lignin and wood. In many cases, these gene-specific downregulations also affect plant development. Specific combinatorial effects of changing the expression of multiple genes are necessary for trait modifications to alleviate negative effects on plant growth and adaptation. Our integrative analysis enables strategic designs for such combinatorial effects. The analysis has also indicated significant novel regulatory components that have not yet been investigated, such as where transcripts and proteins are not well correlated, and epistatic effects imply feed-back regulation. Field testing of the analysis in transgenic Populus will reveal more novel regulation associated with specific growth and adaptation effects that can be incorporated into the engineering designs. Our current integrative analysis is more directly applicable to wood formation in Populus spp. The applicability in other economically important species, such as Eucalypts and pines, remains to be determined. Monolignol biosynthetic pathway genes and metabolic fluxes may vary broadly across genera. With applicable multi-omics data, the integrative analysis approach used here provides a unique strategy that would allow improvements in multiple lignin and wood properties in any woody 
a

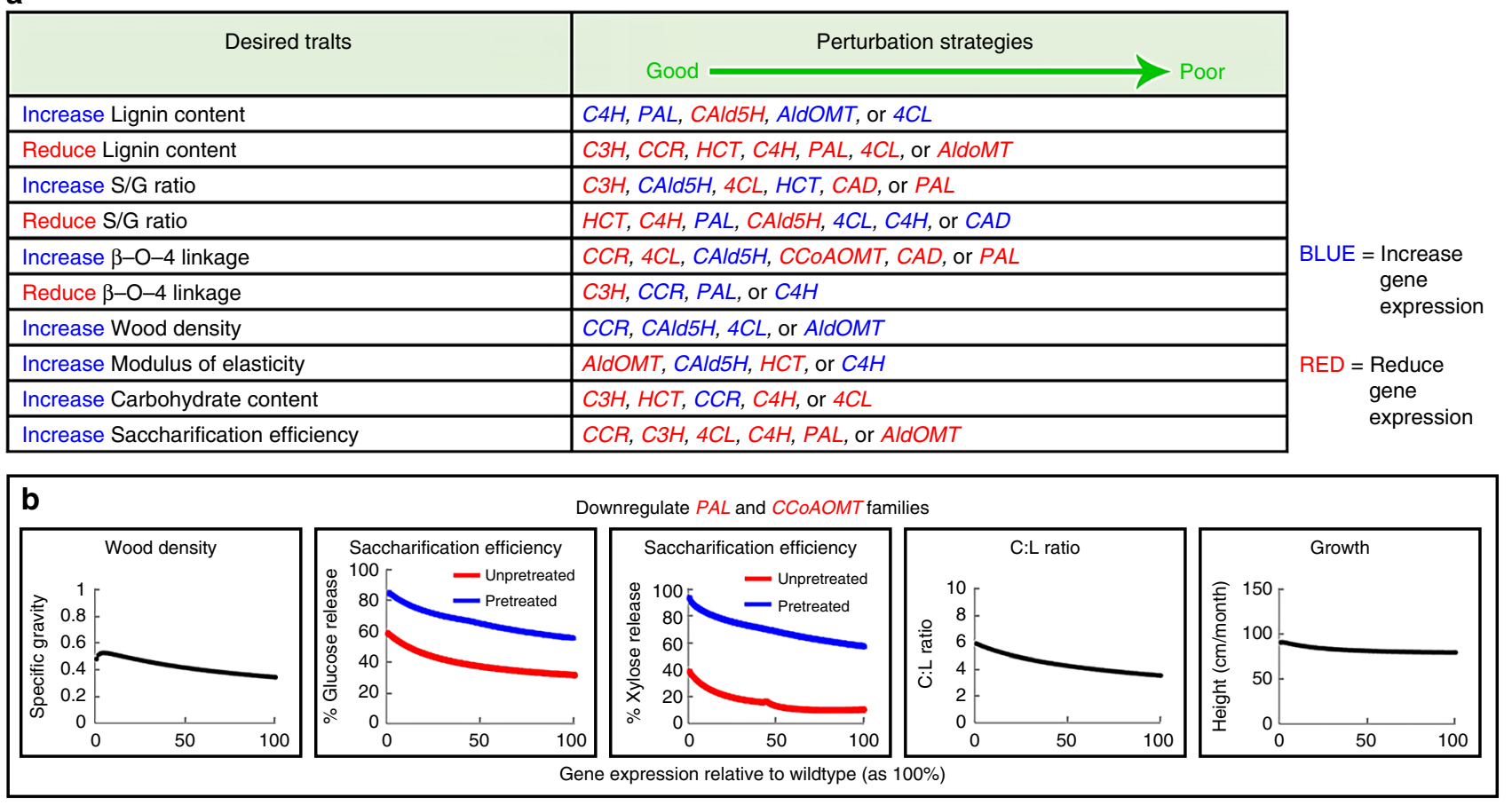

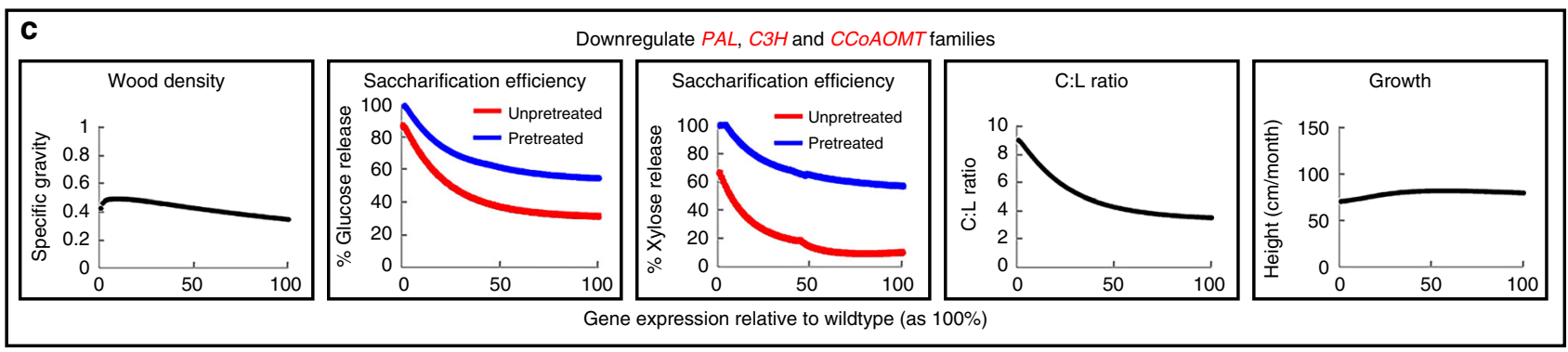

Fig. 10 Strategic engineering of single or combinations of multiple lignin and wood traits through manipulating the expression of specific monolignol genes or combinations of these genes. a The integrative analysis based gene perturbation strategies to achieve specific desired wood traits. Perturbation strategies for each desired trait are arranged from the most significant effector to the least. Blue text represents increased gene expression, and red text represents reduced gene expression. b, c Examples of integrative analysis predicting how to simultaneously improve a combination of three key wood properties without significant impact on plant growth-downregulating two gene families (PtrPAL and PtrCCoAOMT) (b) or downregulating three gene families (PtrPAL, PtrC3H, and PtrCCoAOMT) (c). Each scatterplot is composed of 100 data points, representing 1 to $100 \%$ of wildtype transcript abundance

species. It also provides a general strategy for rigorous definitions of other biological pathways that could lead to a more comprehensive understanding and description of plant metabolism, growth, and adaptation.

\section{Methods}

Plant materials. P. trichocarpa genotype Nisqually-1 was used for all experiments. Wildtype and transgenic trees were grown in $1 / 2$ Miracle-Gro Soil (Scotts MiracleGro products, Maysville, OH, USA) and 1/2 Metro-Mix 200 (Sun Gro, Bellevue, WA, USA) in a greenhouse ( $16 \mathrm{~h}$ light $/ 8 \mathrm{~h}$ dark cycle with supplementary light of $\left.\sim 300 \mu \mathrm{E} \mathrm{m}^{-2} \mathrm{~s}^{-1}\right)^{28}$. Wildtype trees for transformation were 6 months old. All transgenic and the corresponding wildtype trees in soil were grown in a greenhouse for 6 months before harvest for characterization.

Constructs for transgenesis. Gene downregulation constructs were prepared for individual genes, phylogenetic gene-pairs, and gene families. We designed three types of RNAi constructs (types I-III, Supplementary Fig. 1a) and one type of amiRNA construct (type IV, Supplementary Fig. 1a) for these downregulations. Type I RNAi constructs can knock-down individual genes, such as only one xylemspecific family member (e.g., $\mathrm{PtrC} 3 \mathrm{H} 3$ or PtrCCR2) (Supplementary Table 1). Type II RNAi constructs with two cDNA silencing fragments (S1 \& S2) suppress multiple genes. A type III RNAi construct with four cDNA silencing fragments suppress more genes simultaneously. Type IV is an amiRNA construct used to knock- down individual member within gene families that share high sequence similarities ( $>85 \%$ ), such as genes in the $P A L$ and $C 4 H$ families.

Using the downregulation of PtrPAL genes as an example, five amiRNA constructs (Supplementary Table 1) were used to suppress each PtrPAL gene independently. A P. trichocarpa miRNA gene, ptr-MIR408, was used as the transgene to deliver the 21-nt mature amiRNA silencing sequence complementary only to a specific target gene region for transcript cleavage (knock-down). Then a type II RNAi construct (Supplementary Table 1) with two cDNA silencing fragments (S1 \& S2) was designed to suppress the paired PtrPAL1 and 3 genes. Another type II RNAi constructs (Supplementary Table 1) was designed to suppress the group of PtrPAL2, 4, and 5 genes. A type III RNAi construct (Supplementary Table 1) with four cDNA silencing fragments was designed to suppress all five $\operatorname{Ptr} P A L$ genes simultaneously.

All of these constructs contained the $p B I$ binary vector backbone. Its original $35 \mathrm{~S}$ promoter was replaced by the xylem-specific promoter of Ptr4CL3, which we named pBI121-4CLXP plasmid ${ }^{5}$. A xylem-specific promoter ensures that the downstream transgene is specifically expressed in the wood-forming tissue of the transgenic trees. To prepare the gene knockdown constructs, the original GUS sequence of the pBI121-4CLXP plasmid was replaced by an RNAi or amiRNA transgene.

To assemble the RNAi transgene that targets multiple genes, such as genes in the same subgroup or all members of a gene family, one 150-300 bp sequence sharing over $70 \%$ sequence similarity between different target genes but less than $70 \%$ with untargeted genes was selected and used as the RNAi sequence. Such an RNAi sequence fragment was amplified from a cloned gene transcript sequence ${ }^{4}$. If that RNAi sequence did not have sufficient similarity to all target genes $(>70 \%)$, 
more than one similar sequence was prepared and then assembled by overlapping PCR to form the type II and type III RNAi transgenes.

All RNAi fragments were amplified using primers with $3^{\prime}$ sequences that contained appropriate restriction sites. The amplified fragments were digested with restriction enzymes and assembled with a 600 bp GUS linker sequence (GL) to form an antisense: GL: sense fragment as an RNAi transgene sequence and cloned into an intermediate plasmid ${ }^{29}$. The assembled RNAi transgene fragments, confirmed by sequencing, were subcloned into the pBI121-4CLXP plasmid to replace the original GUS sequence ${ }^{5}$.

The amiRNA transgenes were prepared based on the Ptr-MIR408 transcript. The original miRNA408 and miRNA408* sequences were replaced by designed amiRNA and amiRNA* sequences using overlapping $\mathrm{PCR}^{4}$. All amiRNA sequences were designed using the online program WMD (http://wmd.weigelworld.org) ${ }^{30}$ based on the genome of P. trichocarpa v1.0 (JGI). The amiRNA sequences were designed using the "no off-target" option, i.e., the amiRNA had only one specific binding site in the target gene and avoided any possible off-target sites in other genes. AmiRNA* sequences and primer sets for integrating amiRNA and amiRNA* sequences into an amiRNA gene backbone were designed using the WMD program's oligo function under the vector option RS3000 (MIR319a). To integrate the designed amiRNA and amiRNA* sequences into the Ptr-MIR408 transgene backbone, primer sets were modified by replacing the vector sequence of AthMIR319a (RS300 vector) with that of Ptr-MIR408. pBI121-based amiRNA expression binary vectors were assembled following Shi et al. $(2010)^{31}$, except that the promoter-specific forward primer was designed for the xylem-specific promoter

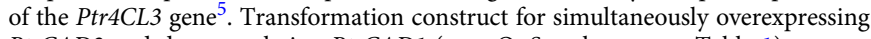
PtrCAD2 and downregulating PtrCAD1 (type O, Supplementary Table 1) was prepared by the insertion of full-length PtrCAD2 coding sequence after the 4CLXP, and the cassette containing 4CLXP-PtrCAD2-NosT was inserted into the PtrCAD1 (type I) construct. All binary constructs were introduced into Agrobacterium tumefaciens C58 for plant transformation ${ }^{28}$.

Transgenic plant production. P. trichocarpa genotype Nisqually-1 was transformed to downregulate genes for monolignol biosynthesis by Agrobacterium transformation ${ }^{28}$ and RNAi or amiRNA constructs (Supplementary Fig. 1a) ${ }^{30}$ listed in Supplementary Table 1. To confirm positive transgene insertions, genomic DNA of putative plantlets was extracted from young leaf tissues using the Qiagen DNeasy Plant Mini Kit (Invitrogen/Life Technologies, Grand Island, NY). PCR assays using promoter forward primers and transgene-specific reverse primers were conducted for the putatively transformed plantlet along with a positive control, the transformation vector plasmid DNA, and a negative control, the genomic DNA from a wildtype tree. For each construct, we produced $\sim 15$ independent transgenic lines. Two vegetatively propagated copies of each line were grown in a greenhouse, and 6-month-old trees were analyzed by qRT-PCR to quantify the levels of target gene knock-down. To select transgenic lines that exhibit different levels of knockdown gene expression, stem-differentiating xylem tissues of transgenic lines of 6months-old greenhouse-grown trees were collected for RNA extraction. Stemdifferentiating xylem was scraped from the surface of the debarked stems using single-edge razor blades ${ }^{22,33}$. The collected tissues were frozen and ground into powder in liquid nitrogen. Total RNA was extracted using the Qiagen RNeasy Mini Kit (Invitrogen/Life Technologies, Grand Island, NY). Target gene transcript abundance was analyzed using qRT-PCR as in our previous studies ${ }^{4}$. Three to fourteen independent transgenic lines were selected per construct to represent varying levels of target gene expression. The selected lines were propagated to 9-15 copies each in the greenhouse. In this way, we produced $\sim 2000$ transgenic $P$. trichocarpa trees for our integrative analysis. All transgenic trees were grown in the greenhouse along with wildtype controls under the same condition ${ }^{29}$. The same tissue and wood samples for each transgenic line and wildtype were used for all analyses (RNA, protein, and wood) (sections below).

Six-months-old trees were harvested for characterization. For each line, three biological replicates were collected; each replicate was a pool of 3-5 clonally propagated trees. Fresh stem-differentiating xylem tissues were collected and stored in liquid nitrogen for analysis by RNA-seq and absolute protein quantification. The corresponding wood samples were collected for quantification of MOE, wood density, lignin content and composition, 2D NMR, wood composition and saccharification efficiency. The transgenic trees were produced and harvested in batches at different times each with wildtype controls, which were used to normalize data collected from different batches (Supplementary Note).

Quantitative estimation of transcript abundance by RNA-seq. Total RNA from each replicate pool (containing 3-5 clonally propagated trees) was extracted using the RNeasy standard protocol (Qiagen, Valencia, CA). Each RNA sample was tested for concentration and purity using a NanoDrop spectrophotometer (Thermo Scientific, Wilmington, DE). The preparation of RNA libraries for sequencing followed Li et al. (2012) ${ }^{34}$, using the TruSeq RNA sample preparation kit (Illumina, San Diego, CA). The RNA libraries were pooled for multiplex sequencing using the Illumina GAIIx platform (Genomic Sciences Laboratory, North Carolina State University, Raleigh, NC). We mapped the FASTQ files from the Illumina GAIIx onto the genome of $P$. trichocarpa $\mathrm{v} 3.0^{3,35-37}$, using Bowtie ${ }^{38}$ and Tophat $^{39}$. BAM (Binary Sequence Alignment/Map) files were converted to raw counts using BedTool $^{40}$, and normalized read counts were obtained using the Trimmed Means of $M$ values $^{35-37,41}$
Protein quantification by PC-IDMS. Total protein extracts were isolated from stem-differentiating xylem and processed using filter-aided sample preparation ${ }^{9}$. The absolute protein abundance of the monolignol biosynthetic enzymes was measured by PC-IDMS using a C18 column $(75 \mu \mathrm{m} \times 15 \mathrm{~cm})$, reversed phase nanoLC and selected reaction monitoring on a TSQ triple quadrupole mass spectrometer (Thermo Scientific, San Jose, CA) ${ }^{9}$. The spectral data was processed and integrated using Skyline ${ }^{42}$ to determine the absolute quantities of proteins in stem-differentiating xylem.

\section{Wood chemistry analysis of transgenics and wildtype. After stem-}

differentiating xylem tissue collection for RNA and protein isolation, the remaining wood stem segments (devoid of internodes 1-5) from the same transgenic and wildtype trees were used for wood chemistry analysis. The stem segments were extracted with $90 \%$ acetone for $48 \mathrm{~h}$, followed by three additional extractions (each $48 \mathrm{~h}$ ) using $100 \%$ acetone, and air-dried. The extractive-free stem segments were ground to a fine powder using a Wiley mill and sieved to 40-60 mesh and vacuum dried over $\mathrm{P}_{2} \mathrm{O}_{5}$. Acid-insoluble lignin and acid-soluble lignin contents were determined following the Klason procedure ${ }^{43}$. Sugars in the acid-soluble lignin fractions were quantified by a gas chromatography-flame ionization detector (GCFID 7890A; Agilent, Santa Clara, CA ${ }^{43}$, or neutralized using $\mathrm{CaCO}_{3}$, filtered through a $0.2 \mu \mathrm{m}$ PVDF membrane (Pall Corporation, Port Washington, NY), and analyzed by an Infinity 1200 HPLC (Agilent, Santa Clara, CA). Pure compounds of glucose, galactose, xylose, mannose, and arabinose (Sigma, St. Louis, MO) were used as standards. The sum of lignin and sugar contents averages $91.6 \%(\%=\mathrm{g} /$ $100 \mathrm{~g}$ dry wood) for wildtype trees; the uronic acid and acetyl contents in wood likely account for much of the remainder.

Quantification of lignin composition and interunit linkages. To ensure that we analyzed essentially the entire lignin fraction of the wood, we utilized nothing more than enzymatic saccharification and did not attempt to further purify the lignin, utilizing the power of the whole-cell-wall 2D NMR methods ${ }^{44,45}$ to resolve components and extract data. To enrich lignin from the wood samples of transgenics and wildtype for 2D NMR analysis, extractive-free wood samples were ground to 40-60 mesh and vacuum dried under $\mathrm{P}_{2} \mathrm{O}_{5}$. The wood samples were then milled using a Pulverisette 7 Planetary Micro Mill (Fritsch, Idar-Oberstein, Germany) at $600 \mathrm{rpm}$, with six cycles of $30 \mathrm{~min}$ on and $15 \mathrm{~min}$ off. The milled samples were incubated with cellulase (Sigma C9422, St. Louis, MO) in the ratio of $1 \mathrm{~g}$ wood per 450 units of cellulase, for $48 \mathrm{~h}$ at $48^{\circ} \mathrm{C}$. The samples were washed twice with an acetate buffer ( $\mathrm{pH} 4.5,20 \mathrm{mM}$ ), followed by two more washes using distilled water, and were freeze dried. The lignin samples were analyzed without further purification to retain the entire lignin without fractionation. The lignin samples were dissolved in DMSO- $\mathrm{d}_{6} /$ pyridine- $_{5}(4: 1, \mathrm{v} / \mathrm{v})$, then analyzed by a $700 \mathrm{MHz}$ Bruker NMR instrument fitted with a cryoprobe for improved sensitivity ${ }^{44}$. S, G, H, and $p$ hydroxybenzoic acid levels were determined by integrating the $\mathrm{H} 2 / \mathrm{C} 2$ correlations using Bruker's Topspin 3.5 software (and expressing on an $\mathrm{S}+\mathrm{G}+\mathrm{H}=100 \%$ basis); relative interunit linkage levels were from integrating $\mathrm{H} \alpha / \mathrm{Ca}$ correlations and are expressed on the basis of the sum of the $(\beta-O-4)+(\beta-5)+(\beta-\beta)+(\beta-1)$ levels $^{45}$.

Quantification of wood modulus of elasticity. Three $20-\mathrm{cm}$ long stem sections were cut from the base of each transgenic and wildtype sample. The stem sections were kept in sealed plastic bags to prevent drying. The stem sections were cut to a length-to-width ratio of 16:1 and analyzed using a three-point bending test ${ }^{46}$ by a universal mechanical tester (MTS Insight, Eden Prairie, MN) to measure the MOE.

Quantification of wood density. One-inch thick disks were cut from the base of each stem and were used to measure wood density (specific gravity) of each tree using the water displacement method ${ }^{47}$. Samples were weighed, dried in an oven at $103 \pm 2{ }^{\circ} \mathrm{C}$ overnight and the oven dry mass measured. The density was calculated following the ASTM standards (D2395) ${ }^{47}$.

Wood pretreatment and enzymatic hydrolysis. Extractive-free wood samples from wood chemistry analysis were either (1) subjected to hot-water pretreatment at $180^{\circ} \mathrm{C}$ for $5 \mathrm{~min}$ in 96 -well reactor plates (pretreated, see below), or (2) without pretreatment (unpretreated) prior to enzymatic hydrolysis. Enzymatic hydrolysis of unpretreated and pretreated samples was carried out using a high-throughput pretreatment and incomplete-saccharification hydrolysis technique to allow comparisons ${ }^{48}$. Briefly, $5 \pm 0.3 \mathrm{mg}$ dry extractive-free wood was weighed into custommade 96-well Hastelloy reactors with Mobile Tool Management robotics (FreeSlate, Sunnyvale, CA). The reactor plates were sealed and pretreated $\left(5 \mathrm{~min}\right.$ at $\left.180^{\circ} \mathrm{C}\right)$ after $250 \mu \mathrm{L}$ of water was added to each well. After cooling, $40 \mathrm{~mL}$ of CTec2 cellulase (Novozymes, Franklinton, NC) diluted in $1.0 \mathrm{M}$ citrate buffer $\mathrm{pH} 5.0$ was added to each well to a final loading of $70 \mathrm{mg}$ protein $/ \mathrm{g}$ glucan. For unpretreated samples, CTec2 was added directly to the reactor plates at the same concentrations as the pretreated samples. The reactor plates were sealed, and the enzymatic hydrolysis was performed at $50^{\circ} \mathrm{C}$ for $70 \mathrm{~h}$. The released glucose and xylose in the supernatants were quantified by sugar-specific oxidation-reduction assays ${ }^{48}$. For each independent wood sample, three analytical replicates were performed. Sugar 
release data were reported as weight percentage ( $w$ t\%) of cell wall residues ${ }^{48}$ and then converted to $\%$ of total glucose or xylose content.

Normalizing transcript and protein abundances and regression. We collected stem-differentiating xylem tissues in six separate batches at different times. Each batch of transgenic trees was planted with wildtype controls. We normalized the RNA-seq read counts of specific transcript and the quantities of specific peptides against the average of three replicates of wildtype in each batch (Supplementary Fig. 3). Linear regression for the transcript (predictor) and protein (dependent variable) abundances of the monolignol biosynthetic pathway genes in the stemdifferentiating xylem of wildtype and transgenic $P$. trichocarpa was performed using JMP software (JMP ${ }^{\circ}$, Version 12, SAS Institute Inc., Cary, NC, 1989-2007).

Integrative analysis. The integrative analysis is represented by equations 1 to 84 (Supplementary Data 2), coded in MATLAB (Supplementary Data 9). There are three main components to the integrative analysis (1-3, Fig. 2b): (1) the transcript/ protein equations (2) the mass-balance kinetic equations and (3) the multiple linear regression equations used to predict the phenotypes (Fig. 2c). The integrative analysis is based on wood formation that consists of a mix of different cell types (fiber, vessel, and ray cells).

Transcript/protein equations. The integrative analysis takes the absolute transcript abundances as the input to predict protein abundances using simple linear regressions $\left(\beta_{\mathrm{i}}\right.$ values, Supplementary Table 2$)$. The simple linear regressions were constrained to intercept 0 (i.e., zero transcript $=$ zero protein). The absolute transcript abundances in $\mu \mathrm{M}$ were estimated from the RNA-seq libraries ${ }^{49}$. Briefly, the raw read counts were normalized to reads per kilobase per million mapped reads, and calibrated to $\mu \mathrm{M}$ using monolignol transcript abundances determined from qRT-PCR of wildtype $P$. trichocarpa stem-differentiating $x^{\prime} \mathrm{sem}^{49}$. The predicted protein abundances were used as the input for the mass-balance kinetic equations.

Mass-balance kinetic equations. The mass-balance equations by Wang et al. $(2014)^{5}$ assumed independence between the monolignol enzyme functions ${ }^{5}$. However, we showed that the two Ptr4CL isoforms (Ptr4CL3 and Ptr4CL5, Fig. 1), each with distinct kinetic and inhibitory properties, form a heterotetrameric protein complex in vivo in the ratio of $3: 1^{12}$. This $4 \mathrm{CL}$ protein complex affects the specificity and reaction rates of CoA ligation (fluxes 7 to 11, Fig. 1) ${ }^{12}$, improves the stability/ robustness of the pathway ${ }^{50}$, and constitutes an important component of metabolic regulation ${ }^{12}$. Therefore, we modified the mass-balance equations from Wang et al. $(2014)^{5}$ to include the updated flux equations from Chen et al. $(2014)^{12}$ and Lin et al. $(2015)^{7}$, which incorporate the heterotetrameric 4CL protein complex for fluxes 7 and 8 (Fig. 1), and the associated complex enzyme inhibition kinetics. The modified equations (equations 21 to 59, Supplementary Data 2) were coded into MATLAB 2015b using the ordinary differential equation solver (ODE15s) to predict pathway metabolic-fluxes and metabolite concentrations. We presented evidence that three monolignol cytochrome $\mathrm{P} 450$ monooxygenases ( $\mathrm{PtrC} 4 \mathrm{H} 1$, $\mathrm{PtrC} 4 \mathrm{H} 2$, and PtrC3H3) interact to form heteromeric protein complexes ${ }^{51}$. However, mass-balance kinetic equations that describe these interactions were not part of the integrative analysis because they are difficult to determine, and biochemical techniques are not currently available to assay the membrane-bound enzymes at different molar ratios. To determine the initial conditions, we used MATLAB and equations 21 to 59 to solve for the phenylalanine concentration when wildtype S/G ratio is 2.16 (equivalent to fluxes V35/V34, Fig. 1), a quantity based on our 2D NMR analysis of isolated lignin from the stem-differentiating xylem of wildtype $P$. trichocarpa (Supplementary Data 4). MATLAB shows that a phenylalanine concentration of $1.4 \mu \mathrm{M}$ is necessary to produce a wildtype $\mathrm{S} / \mathrm{G}$ ratio of $\sim 2.16$. The remaining metabolite concentrations vary dynamically in MATLAB until a steady-state is reached. The simulation time was set from 0 to $10,000 \mathrm{~s}$, which provides sufficient time for the metabolic-fluxes and metabolite concentrations to reach steady-state values. We tested 10,000 sets of random initial metabolite concentrations (Latin Hypercube Sampling) to determine all possible steady-states and found $92 \%$ of all simulations settled at the same steady-state, suggesting that the pathway is robust to changing initial conditions. 4-Coumaryl alcohol (20, H-subunit precursor, Fig. 1), coniferyl alcohol (22, G-subunit precursor), and sinapyl alcohol (24, S-subunit precursor) are formulated as precursors of the terminal products $(\mathrm{H}, \overline{\mathrm{G}}$, and S-subunits) that accumulate over the course of the flux analysis.

Multiple linear regressions. Using JMP Pro 12 (SAS Institute Inc., Cary, NC), an allpossible-model approach was used to determine which of the metabolites and metabolic-fluxes are the best linear predictors for each of the lignin and wood properties. The all-possible-model approach tests all combinations of metabolites and fluxes as predictors to identify the best multiple linear regressions (equations 60 to 84, Supplementary Data 2) that exhibit global minima in the corrected Akaike's information criterion (AICc). To reduce multicollinearity, metabolites and fluxes with correlation coefficients $>0.95$ were omitted from the multiple linear regressions. The predicted lignin and wood properties from the multiple linear regressions are restricted to non-negative values, and the properties that have the unit of percentage (e.g., $\beta-O-4$ linkages) are restricted to a maximum value of $100 \%$.

Integrative analysis validation. The integrative analysis was validated using 5fold cross-validation. For the 5 -fold cross-validation, each of the 239 transgenic and wildtype lines was randomly sorted into five approximately equal sized groups. Using JMP Pro 12 (SAS Institute Inc., Cary, NC), an all-possible-model approach was used to determine which of the metabolites and fluxes were the best linear predictors for each group, based on the lowest AICc value. The all-possible-model algorithm re-estimates all regression parameters including the transcript/protein $\beta_{\mathrm{i}}$ values and the multiple linear regression constants. To prevent the algorithm from choosing fluxes and metabolites with very small values and assigning them a very large scaling factor, the metabolite concentrations and fluxes were rounded to five decimal places for this process. The predictors that yield the lowest AICc value were chosen to be included in the integrative analysis (Supplementary Data 2).

Direct validation of metabolite concentrations and metabolic-fluxes derived from the mass-balance equations (equations 21-59, Supplementary Data 2) is not possible because there is no reliable extraction technique that permits the quantitation isolation of monolignol pathway metabolites. We have investigated extensively the quantification of monolignol pathway metabolites in vivo, by synthesizing stable-isotope-labeled standard compounds and using advanced LCMS/MS systems. Our analysis confirmed that monolignol pathway metabolites could not be reliably extracted from wood forming tissues for quantification. Similarly, metabolic-fluxes for monolignol biosynthesis cannot be validated experimentally, due to the complex enzyme inhibitory network that regulates the pathway. These technical difficulties have long prohibited the use of metabolite concentrations and metabolic-fluxes for experimental validation. The most direct means of validating the integrative analysis is by association to the content, composition, and linkage structures of lignin, which we applied in our five-fold cross-validation. Cross-validation is suitable here because its re-sampling technique allows for efficient use of all available data and provides a robust estimation of the validity of predictions with minimal bias and variability. Considering the integrative analysis is based on $\sim 2000$ transgenic trees, a small independent experiment would be insufficient to provide an adequate validation.

Genetic engineering strategies for optimal wood properties. To identify the optimal approaches for improving any single or combination of lignin and wood properties, we used the integrative analysis to investigate all possible combinations of monolignol gene perturbations. Each gene family transcript abundance was either (1) upregulated to $1000 \%$ of the wildtype level, (2) retained at the wildtype level, or (3) downregulated to $5 \%$ of the wildtype level. Gene upregulation by $1000 \%$ is an estimate of the level of gene-specific overexpression feasible by transgenesis, and represents an extrapolated projection of how increasing monolignol gene expression affects lignin and wood traits. Such extrapolation is outside of the experimental data and should be interpreted with caution. In silico gene perturbations were limited to a simultaneous modification of one, two, or three gene families. Untargeted gene families were set at the wildtype level because indirect effects were not included in the integrative analysis equations (Supplementary Data 2). A total of 1161 unique combinations of monolignol gene family perturbations were tested using the integrative analysis (equations 1 to 84, Supplementary Data 2). The predicted lignin and wood properties were then individually ranked from the highest value to the lowest, or vice versa, depending on the desired traits. The gene perturbations that produced the most desired traits were reported in Fig. 10a. For maximal improvement of multiple lignin and wood properties, the best perturbation approaches were identified by ranking each predicted outcome based on the extent of the overall improvement over wildtype for the specific desired properties. To do this, we applied proportional scoring ${ }^{52}$ to each predicted outcome using the equation:

$$
\sum_{i=1}^{N}\left(\text { predicted property }_{i}-\text { desired property }{ }_{i}\right)^{2}
$$

Where $N$ represents the number of desired properties. The scores were then ranked from the lowest value (most desired outcome) to the highest (least desired outcome) to identify the best perturbation approaches (Fig. 10b, c).

Data availability. The RNA-seq libraries are available under GEO accession number GSE78953. Proteomics, MOE, wood density, 2D NMR, plant growth, and wood chemistry data sets are available on CyVerse [http://mirrors. iplantcollaborative.org/browse/iplant/home/shared/LigninSystemsDB]. Correspondence and requests for materials should be addressed to V.L.C. (vchiang@ncsu. edu).

Received: 14 July 2017 Accepted: 16 March 2018 Published online: 20 April 2018 


\section{References}

1. Payen, A. Recherches sur la matière incrustante des bois. Acad. Sci. Paris Compt. Rend. VIII, 169-170 (1839).

2. Freudenberg, K. Lignin: Lignin: Its constitution and formation from $p$ hydroxycinnamyl alcohols: Lignin is duplicated by dehydrogenation of these alcohols; intermediates explain formation and structure. Science 148, 595-600 (1965).

3. Tuskan, G. A. et al. The genome of black cottonwood, Populus trichocarpa (Torr. \& Gray). Science 313, 1596-1604 (2006).

4. Shi, R. et al. Towards a systems approach for lignin biosynthesis in Populus trichocarpa: Transcript abundance and specificity of the monolignol biosynthetic genes. Plant Cell Physiol. 51, 144-163 (2010).

5. Wang, J. P. et al. Complete proteomic-based enzyme reaction and inhibition kinetics reveal how monolignol biosynthetic enzyme families affect metabolicflux and lignin in Populus trichocarpa. Plant Cell 26, 894-914 (2014).

6. Chen, H. et al. Monolignol pathway 4-coumaric acid:coenzyme A ligases in Populus trichocarpa: novel specificity, metabolic regulation, and simulation of coenzyme A ligation fluxes. Plant Physiol. 161, 1501-1516 (2013).

7. Lin, C. Y. et al. 4-Coumaroyl and caffeoyl shikimic acids inhibit 4-coumaric acid:coenzyme A ligases and modulate metabolic-flux for 3-hydroxylation in monolignol biosynthesis of Populus trichocarpa. Mol. Plant 8, 176-187 (2015).

8. Wang, J. P. et al. Functional redundancy of the two 5-hydroxylases in monolignol biosynthesis of Populus trichocarpa: LC-MS/MS based protein quantification and metabolic-flux analysis. Planta 236, 795-808 (2012)

9. Shuford, C. M. et al. Comprehensive quantification of monolignol-pathway enzymes in Populus trichocarpa by protein cleavage isotope dilution mass spectrometry. J. Proteome Res. 11, 3390-3404 (2012).

10. Lu, P., Vogel, C., Wang, R., Yao, X. \& Marcotte, E. M. Absolute protein expression profiling estimates the relative contributions of transcriptional and translational regulation. Nat. Biotechnol. 25, 117-124 (2007).

11. Milo, R., Jorgensen, P., Moran, U., Weber, G. \& Springer, M. BioNumbersthe database of key numbers in molecular and cell biology. Nucleic Acids Res. 38, D750-D753 (2010).

12. Chen, H. et al. Systems biology of lignin biosynthesis in Populus trichocarpa: Heteromeric 4-coumaric acid:coenzyme A ligase protein complex formation, regulation, and numerical modeling. Plant Cell 26, 876-893 (2014).

13. $\mathrm{Hu}, \mathrm{W}$. J. et al. Repression of lignin biosynthesis promotes cellulose accumulation and growth in transgenic trees. Nat. Biotechnol. 17, 808-812 (1999).

14. Voelker, S. L., Lachenbruch, B., Meinzer, F. C., Kitin, P. \& Strauss, S. H. Transgenic poplars with reduced lignin show impaired xylem conductivity, growth efficiency and survival. Plant Cell Environ. 34, 655-668 (2011).

15. Bonawitz, N. D. et al. Disruption of mediator rescues the stunted growth of a lignin-deficient Arabidopsis mutant. Nature 509, 376-380 (2014).

16. Gallego-Giraldo, L., Escamilla-Trevino, L., Jackson, L. A. \& Dixon, R. A. Salicylic acid mediates the reduced growth of lignin down-regulated plants. Proc. Natl. Acad. Sci. USA 108, 20814-20819 (2011).

17. Zobel, B. J. \& Jett, J. B. Genetics of Wood Production (Springer-Verlag, Berlin, Heidelberg, 1995).

18. Zhang, S. Y. Wood specific gravity-mechanical property relationship at species level. Wood Sci. Technol. 31, 181-191 (1997)

19. Yang, B. \& Wyman, C. E. Pretreatment: the key to unlocking low-cost cellulosic ethanol. Biofuel Bioprod. Biorefin. 2, 26-40 (2008).

20. Min, D., Li, Q., Jameel, H., Chiang, V. \& Chang, H. M. The cellulase-mediated saccharification on wood derived from transgenic low lignin lines of black cottonwood (Populus trichocarpa). Appl. Biochem. Biotechnol. 168, 947-955 (2012).

21. Chen, F. \& Dixon, R. A. Lignin modification improves fermentable sugar yields for biofuel production. Nat. Biotechnol. 25, 759-761 (2007).

22. Van Acker, R. et al. Lignin biosynthesis perturbations affect secondary cell wall composition and saccharification yield in Arabidopsis thaliana. Biotechnol. Biofuels 6, 46-46 (2013).

23. Vanholme, R. et al. Caffeoyl shikimate esterase (CSE) is an enzyme in the lignin biosynthetic pathway in Arabidopsis. Science 341, 1103-1106 (2013).

24. Ha, C. M. et al. An essential role of caffeoyl shikimate esterase in monolignol biosynthesis in Medicago truncatula. Plant. J. 86, 363-375 (2016).

25. Vargas, L. et al. Improving total saccharification yield of Arabidopsis plants by vessel-specific complementation of caffeoyl shikimate esterase (cse) mutants. Biotechnol. Biofuels 9, 139 (2016).

26. Porth, I. et al. Populus trichocarpa cell wall chemistry and ultrastructure trait variation, genetic control and genetic correlations. New. Phytol. 197, 777-790 (2013).

27. Bhagia, S., Muchero, W., Kumar, R., Tuskan, G. A. \& Wyman, C. E. Natural genetic variability reduces recalcitrance in poplar. Biotechnol. Biofuels 9, 106 (2016).

28. Song, J., Lu, S., Chen, Z., Lourenco, R. \& Chiang, V. L. Genetic transformation of Populus trichocarpa genotype Nisqually-1: A functional genomic tool for woody plants. Plant Cell Physiol. 47, 1582-1589 (2006).
29. Li, Q. et al. Down-regulation of glycosyltransferase 8D genes in Populus trichocarpa caused reduced mechanical strength and xylan content in wood. Tree Physiol. 31, 226-236 (2011).

30. Schwab, R., Ossowski, S., Riester, M., Warthmann, N. \& Weigel, D. Highly specific gene silencing by artificial microRNAs in Arabidopsis. Plant Cell 18, 1121-1133 (2006).

31. Shi, R., Yang, C., Lu, S., Sederoff, R. \& Chiang, V. L. Specific down-regulation of PAL genes by artificial microRNAs in Populus trichocarpa. Planta 232, 1281-1288 (2010)

32. Lin, Y. et al. A simple improved-throughput xylem protoplast system for studying wood formation. Nat. Protoc. 9, 2194-2205 (2014).

33. Li, W. et al. A robust chromatin immunoprecipitation protocol for studying transcription factor-DNA interactions and histone modifications in woodforming tissue. Nat. Protoc. 9, 2180-2193 (2014).

34. Li, Q. et al. Splice variant of the SND1 transcription factor is a dominant negative of SND1 members and their regulation in Populus trichocarpa. Proc. Natl. Acad. Sci. USA 109, 14699-14704 (2012).

35. Shi, R. et al. Tissue and cell-type co-expression networks of transcription factors and wood component genes in Populus trichocarpa. Planta 245, 927-938 (2017).

36. Wang, J. P. et al. Phosphorylation is an on/off switch for 5hydroxyconiferaldehyde O-methyltransferase activity in poplar monolignol biosynthesis. Proc. Natl. Acad. Sci. USA 112, 8481-8486 (2015).

37. Lin, Y. J. et al. Reciprocal cross-regulation of VND and SND multigene TF families for wood formation in Populus trichocarpa. Proc. Natl. Acad. Sci. USA 114, E9722-E9729 (2017)

38. Langmead, B., Trapnell, C., Pop, M. \& Salzberg, S. L. Ultrafast and memoryefficient alignment of short DNA sequences to the human genome. Genome Biol. 10, R25 (2009)

39. Kim, D. et al. TopHat2: accurate alignment of transcriptomes in the presence of insertions, deletions and gene fusions. Genome Biol. 14, R36 (2013).

40. Quinlan, A. R. \& Hall, I. M. BEDTools: a flexible suite of utilities for comparing genomic features. Bioinformatics 26, 841-842 (2010)

41. Robinson, M. D. \& Oshlack, A. A scaling normalization method for differential expression analysis of RNA-seq data. Genome Biol. 11, R25 (2010).

42. MacLean, B. et al. Skyline: an open source document editor for creating and analyzing targeted proteomics experiments. Bioinformatics 26, 966-968 (2010).

43. $\mathrm{Lu}, \mathrm{S}$. et al. Ptr-miR397a is a negative regulator of laccase genes affecting lignin content in Populus trichocarpa. Proc. Natl. Acad. Sci. USA 110, 10848-10853 (2013).

44. Kim, H. \& Ralph, J. Solution-state 2D NMR of ball-milled plant cell wall gels in DMSO- $d_{6} /$ pyridine- $d_{5}$. Org. Biomol. Chem. 8, 576-591 (2010).

45. Mansfield, S. D., Kim, H., Lu, F. \& Ralph, J. Whole plant cell wall characterization using solution-state 2D NMR. Nat. Protoc. 7, 1579-1589 (2012).

46. Horvath, B., Li, L., Kasal, B., Peralta, P. \& Peszlen, I. Effect of lignin genetic modification on wood anatomy of aspen trees. IAWA J. 31, 29-38 (2010).

47. ASTM International. Annual Book of ASTM Standards, Vol. 4.10 (ASTM International, West Conshohocken, PA, 2009).

48. Decker, S. R., Brunecky, R., Tucker, M. P., Himmel, M. E. \& Selig, M. J. High throughput screening techniques for biomass conversion. Bioenerg. Res. 2, 179-192 (2009).

49. Shi, R. et al. Regulation of phenylalanine ammonia-lyase $(P A L)$ gene family in wood forming tissue of Populus trichocarpa. Planta 238, 487-497 (2013).

50. Naik, P. P. A Systems Biology Approach Towards Understanding the Regulation of Monolignol Biosynthesis in Populus trichocarpa. PhD dissertation, North Carolina State Univ. (2016).

51. Chen, H. C. et al. Membrane protein complexes catalyze both 4- and 3hydroxylation of cinnamic acid derivatives in monolignol biosynthesis. Proc. Natl. Acad. Sci. USA 108, 21253-21258 (2011).

52. Hair, J. F., Anderson, R. E., Tatham, R. L. \& Black, W. C. Multivariate Data Analysis (Prentice-Hall, Upper Saddle River, NJ, 1998).

\section{Acknowledgements}

This work was supported by the National Natural Science Foundation of China (NSFC) Grants 31430093, 31470672, and 31522014. We also thank the financial support from the U.S. National Science Foundation, Plant Genome Research Program Grant DBI-0922391, the NC State University Jordan Family Distinguished Professor Endowment, and the NC State University Forest Biotechnology Industrial Research Consortium. H.K. and J.R., and the NMR use were supported in part by the DOE Great Lakes Bioenergy Research Center (DOE BER Office of Science DE-FC02-07ER64494). The saccharification assay used in this study was developed as part of the BioEnergy Science Center. The BioEnergy Science Center is a U.S. Department of Energy Bioenergy Research Center supported by the Office of Biological and Environmental Research in the DOE Office of Science. 


\section{Author contributions}

J.P.W., R.S., C.Y., H.-C.C., S.T.-A., H.C., H.-S.S., Y.-C.L., W.L. and Q.L. generated the transgenics and measured the plant growth. R.S., Q.L. and Y.-H.S. performed the RNA-seq. J.P.W., J.L., C.-Y.L., C.M.S., D.C.M. and P.L.L. carried out the proteomics. J.P.W., C.Y.L., H.-M.C. J.L. and T.-F.Y. measured the wood chemistry. J.L., H.K. and J.R. performed and analyzed the 2D NMR. I.P., Z.M. and C.W.E. determined the MOE. J.P.W., I.P., Z.M. and C.W.E. measured the wood density. E.G., T.S. and M.F.D. performed the wood saccharification. J.P.W., M.L.M., C.M.W., J.J.D., J.S., P.N., Y.S., B.G.L., C.S., Y.-Y.H. and F.I. assembled the integrative equations and performed the simulations. J.P.W., M.L.M., C.M. W., J.J.D., R.R.S. and V.L.C. developed the concept of the article and wrote the paper.

\section{Additional information}

Supplementary Information accompanies this paper at https://doi.org/10.1038/s41467018-03863-z.

Competing interests: The authors declare no competing interests.

Reprints and permission information is available online at http://npg.nature.com/ reprintsandpermissions/
Publisher's note: Springer Nature remains neutral with regard to jurisdictional claims in published maps and institutional affiliations.

(c) (i) Open Access This article is licensed under a Creative Commons Attribution 4.0 International License, which permits use, sharing, adaptation, distribution and reproduction in any medium or format, as long as you give appropriate credit to the original author(s) and the source, provide a link to the Creative Commons license, and indicate if changes were made. The images or other third party material in this article are included in the article's Creative Commons license, unless indicated otherwise in a credit line to the material. If material is not included in the article's Creative Commons license and your intended use is not permitted by statutory regulation or exceeds the permitted use, you will need to obtain permission directly from the copyright holder. To view a copy of this license, visit http://creativecommons.org/ licenses/by/4.0/.

(C) The Author(s) 2018 